\title{
The robust machine availability problem - bin packing under uncertainty
}

\author{
Guopeng Song, Daniel Kowalczyk, Roel Leus \\ ORSTAT, Faculty of Economics and Business, KU Leuven, Belgium \\ E-mail: firstname.lastname@kuleuven.be
}

\begin{abstract}
We define and solve the robust machine availability problem in a parallel machine environment, which aims to minimize the number of identical machines required while completing all the jobs before a given deadline. The deterministic version of this problem essentially coincides with the bin packing problem. Our formulation preserves a user-defined robustness level regarding possible deviations in the job durations. For better computational performance, a branch-andprice procedure is proposed based on a set covering reformulation. We use zero-suppressed binary decision diagrams for solving the pricing problem, which enable us to manage the difficulty entailed by the robustness considerations as well as by extra constraints imposed by branching decisions. Computational results are reported that show the effectiveness of a pricing solver with zero-suppressed binary decision diagrams compared with a mixed integer programming solver.
\end{abstract}

Keywords: parallel machine scheduling, machine availability, bin packing, robust optimization, branch and price, ZDD

\section{Introduction}

Optimization problems arising in planning and scheduling are often subdivided into two categories (see, for instance, Möhring, 1984). In resource-driven problems, the resource availability is constrained and the objective is to schedule all the jobs as well as possible within the resource limits, for instance with minimum makespan. In time-driven problems, on the other hand, a fixed time horizon is given and the aim is to minimize the costs of the capacity required to perform all the work 
within the horizon. Whereas most of the operational scheduling literature has been written for the first case (see Pinedo, 2015, for an extensive overview), there are also situations where resources can be acquired or released in practically any desired amount if one is willing to pay the expenses involved in changing resource levels, such as the costs of hiring, training, unemployment insurance, and so on (Wiest et al., 1969). This setting is in line with the chase strategy in aggregate planning (Chopra and Meindl, 2013), where the key lever for synchronizing production and demand is the short-term variation of capacity and workforce. Similar models have been built in the context of rough-cut capacity planning for project-based environments (also called the resource loading problem), where the focus is on matching production capacity and customer demand while minimizing the cost of the use of nonregular capacity as well as customer order tardiness (Hans, 2001, Wullink et al. 2004). Time-driven problems have also received attention in the operational scheduling literature, in particular under the form of the resource availability cost problem (Möhring, 1984, Demeulemeester, 1995; Rodrigues and Yamashita, 2010; Coughlan et al., 2015), where a deadline for the job set is given and the cost of the required resources is to be minimized. The closely related resource leveling problem seeks to complete the work within the given due date and keep the resource usage as leveled as possible (Easa, 1989; Harris, 1990; Leu et al., 2000).

In practical planning and scheduling, uncertainty is inevitable. The uncertainty can originate both from environment factors and system factors (Mula et al., 2006), and may cause significant disturbances during the execution of pre-computed plans or schedules (Herroelen and Leus, 2005). Thus it is desired to develop methods that either can reflect the uncertain nature of the available information or provide some guarantee regarding the insensitivity of the solution to the information unfolded (Graves, 1981). In the parallel machine scheduling environment, most of the uncertain factors can be modeled into the jobs' processing times (Pinedo, 2015), which lays the groundwork for the construction of practical optimization models under uncertainty. There are two general paradigms for incorporating knowledge of uncertainty into a schedule, namely stochastic programming and robust optimization. Stochastic programming works with a probabilistic description of the uncertainty, in other words, the probability distributions of the uncertain parameters. Accurate probability distributions are not easy to acquire in practice. Stochastic programming usually also requires great computational effort. Robust optimization, on the other hand, can still work with incomplete probabilistic information, and is typically more tractable from a practical point of view 
(e.g., Bertsimas and Thiele, 2006; Bertsimas et al., 2011).

Parallel machine scheduling has been studied for many decades. The most classic objective is makespan minimization; the resulting problem is usually denoted as $P \| C_{\max }$ in the classic threefield notation, and is known to be NP-hard (Garey and Johnson, 1979). In this paper, the robust machine availability problem (RMAP) is addressed in a parallel machine environment. The goal is to minimize the required number of identical machines and complete all the jobs before a common deadline, when the job processing times are uncertain. The deterministic variant of this problem corresponds to the classic bin packing problem (BPP). We study a robust reformulation based on a convex uncertainty set as proposed by Bertsimas and Sim (2004). With a budget for the number of jobs that can deviate from their nominal processing times, the model provides a trade-off between cost and robustness while preserving linearity.

Our main contributions can be described as follows. To the best of our knowledge, the RMAP has not yet been studied before, and through the link with the BPP we are also one of the first to study robust bin packing. This work also constitutes a possible starting point for a solution method for a robust version of $P \| C_{\max }$, which could be solved using RMAP as a subproblem in a binary search procedure, similarly to Dell'Amico et al. (2008). For enhanced computational performance, we propose a branch-and-price $(\mathrm{B} \& \mathrm{P})$ procedure based on a set covering reformulation, in which we apply the branching scheme of Ryan and Foster (1981). The duration uncertainty and the branching constraints render the pricing problem very difficult, so that it cannot be effectively handled by a general mixed integer programming (MIP) solver. We therefore apply zero-suppressed binary decision diagrams (ZDDs) for solving the pricing problem; these ZDDs provide a compact representation of the solution space and allow for efficient basic set operations to append branching constraints.

The remainder of this paper is organized as follows. Section 2 contains an extensive literature review. In Section 3 we present a formal problem statement of the RMAP, for which a set covering reformulation leading to a B\&P procedure is described in Section 4. Section 5 applies ZDDs for solving the pricing problem in the $\mathrm{B} \& \mathrm{P}$ procedure. In Section 6, computational results are reported that show the effectiveness of the proposed algorithm, along with a sensitivity analysis of the influence of the uncertainty budget. We provide a summary and conclusions in Section 7 . 


\section{Literature review}

In this section we provide a brief survey of recent work on the related subjects of parallel machine scheduling, bin packing, robust optimization and robust scheduling.

Parallel machine scheduling A large body of literature exists on scheduling identical machines in parallel. Dell'Amico and Martello (1995) propose a branch-and-bound (B\&B) algorithm for $P \| C_{\max }$, using tight bounds based on the relationship with the BPP. Mokotoff $(2004)$ uses a MIP formulation with a cutting-plane method to optimally solve $P \| C_{\max }$. Comparative experiments are carried out by Dell'Amico and Martello (2005), showing that their 1995-algorithm consistently outperforms the method of Mokotoff (2004). Dell'Amico et al. (2008) propose a heuristic method for solving $P \| C_{\max }$ based on scatter search, followed by an exact binary search procedure with integrated $\mathrm{B} \& \mathrm{P}$ scheme that iteratively solves BPP instances as a subproblem. Van den Akker et al. (1999) investigate parallel machine scheduling to minimize the total weighted completion time. They propose a $\mathrm{B} \& \mathrm{P}$ algorithm based on a set partitioning formulation with the pricing problem solved by a dynamic programming (DP) procedure, and branch on the job completion times such that the structure of the pricing problem is preserved. Chen and Powell (1999) independently propose a column generation (CG) procedure for the same problem, but they branch on the jobs' pairwise precedence relations in order to maintain the structure of the pricing problem. Pessoa et al. (2010) develop an arc-time-indexed formulation for the total weighted tardiness problem on parallel machines, which is solved using a branch-cut-and-price procedure.

Bin packing The one-dimensional BPP can be seen as a "dual" to $P \| C_{\max }$ (Dell'Amico et al. 2008). The BPP coincides with the deterministic machine availability problem on parallel machines (see Section 3.1), where the number of machines (bins) with given time limit (capacity) to execute (fit) all jobs (items) is to be minimized. The BPP is also NP-hard (Garey and Johnson, 1979). The BPP is a variant of the cutting stock problem (CSP); we refer to Delorme et al. (2016) for a recent survey of the BPP and the CSP. To the best of our knowledge, there is no published work on robust bin packing in the mainstream literature, although some research has appeared on bin packing with stochastic item sizes. Rhee and Talagrand (1993) study a bin packing problem where the item sizes are identically distributed according to a given distribution. 
Robust optimization Robust optimization has been studied for decades, and is a valuable methodology when complete probabilistic information is not accessible. One of the earliest works in robust optimization is Soyster (1973), where a reformulation of an original LP model is used to obtain a solution that is resistant to any possible perturbation and feasible for any realization, thus protecting against the worst possible scenario. Kouvelis and $\mathrm{Yu}(1997)$ present the framework of robust discrete optimization, focusing on regret-based objectives under a discrete scenario set. The worst-case method adopted by these references typically produces overly conservative solutions. To accommodate this problem of over-conservatism, Ben-Tal and Nemirovski $(1998,1999,2000)$ and El Ghaoui and Lebret (1997), El Ghaoui et al. (1998) have independently worked on methods that restrict the uncertain parameters to ellipsoidal uncertainty sets, where the robust counterpart is a conic quadratic problem, thus resulting in an increase of complexity. Later on, Bertsimas and Sim (2003, 2004) propose a new robust linear optimization approach based on a polyhedral uncertainty set, where the level of conservatism can be adjusted and the robust counterpart remains a linear problem. The latter method has the advantage of combining flexibility and tractability.

Robust scheduling The idea of minimizing worst-case deviation from optimality has also been applied in machine scheduling problems (Kouvelis et al. 2000$)$. Xu et al. $(2013,2014)$ study the robust parallel machine scheduling problem with interval job processing times, minimizing the maximal regret from the optimal objective value over all possible realizations. Different robust measures have also been defined and applied for generating robust schedules; relevant work includes Daniels and Kouvelis (1995), Yang and Yu (2002), Leus and Herroelen (2004), Kasperski (2005) and Lu et al. (2012). Ranjbar et al. (2012) maximize the probability that a threshold on the makespan is not exceeded in an identical parallel machine environment, where the job processing times are assumed to be normally distributed. Despite the sparse work on robust scheduling, multiple applications of the uncertainty set proposed by Bertsimas and Sim (2004) can be found in the literature - see, for example, Pessoa et al. (2015), Bougeret et al. (2016) and Bruni et al. (2017). 


\section{Problem statement}

In this section we first describe the deterministic variant of the machine availability problem (Section 3.1), followed by the robust machine availability problem RMAP in Section 3.2 .

\subsection{The deterministic machine availability problem}

Given are a job set $J$ containing $n$ jobs, with processing time $p_{j}$ for job $j(j=1, \ldots, n)$, a sufficiently large set $M$ of identical machines (for instance, with $M \geq n$ ), and a deadline $T$ (with all $p_{j} \leq T$ ). The maximum completion time among all jobs is called the makespan. The objective of the machine availability problem is to find the lowest number of machines so that each job can be assigned to a machine and the makespan does not exceed the deadline. As mentioned supra, this problem is equivalent with the BPP. A textbook formulation therefore is the following, in which a binary decision variable $x_{i j}$ is introduced for each machine $i$ and job $j$, taking the value 1 if job $j$ is assigned to machine $i$ and 0 otherwise. The binary variable $y_{i}$ decides whether machine $i$ is used or not.

$$
\begin{aligned}
& \text { minimize } \sum_{i \in M} y_{i} \\
& \text { subject to } \sum_{j \in J} p_{j} x_{i j} \leq T y_{i} \quad \forall i \in M \\
& \sum_{i \in M} x_{i j}=1 \quad \forall j \in J \\
& x_{i j} \in\{0,1\} \quad \forall i \in M, \forall j \in J \\
& y_{i} \in\{0,1\} \quad \forall i \in M
\end{aligned}
$$

The objective function (1a) is to minimize the total number of machines required. The inequalities (1b) state that the work on each machine $i$ cannot exceed the deadline. The constraints (1c) guarantee that each job is assigned to one and only one machine.

\subsection{Robust reformulation}

The risk of over-conservatism is inherent in the robust optimization paradigm, and we apply the modelling choice of Bertsimas and Sim (2004) to adjust the trade-off between cost and robustness, while preserving linearity. For each job $j \in J$, the processing time $p_{j}$ is assumed to belong to a 
symmetric and bounded interval $\left[\bar{p}_{j}-\hat{p}_{j}, \bar{p}_{j}+\hat{p}_{j}\right]$, where $\bar{p}_{j}$ is the nominal or expected value and $\hat{p}_{j}$ is the deviation. Both $\bar{p}_{j}$ and $\hat{p}_{j}$ are non-negative integers, with $\bar{p}_{j}>\hat{p}_{j}$. An integer $\Gamma$ is given as a budget for the number of jobs in $J$ that can deviate from their nominal processing times, which controls the "price" of robustness. If the deadline is violated by any individual machine, the solution is infeasible. A robust solution should then also protect against the extreme situation where all deviating jobs are concentrated on the same machine, and consequently $\Gamma$ is imposed as an upper bound on the number of jobs disrupted on each machine. This coincides with the uncertainty set proposed by Bertsimas and Sim (2004), where the budget for robustness is considered in individual constraints. The disruption budget $\Gamma$ is not a value known or given to the decision maker. Instead, it is a parameter that should be tuned and chosen deliberately: different values correspond with different levels of risk aversion, and can be used to analyze the effect of different levels of robustness in the schedule. It should also be noted that a solution is not only protected from disruption in at most $\Gamma$ jobs per machine. The $\Gamma$ value actually implies a bound on the probability of violation of the deadline constraint (see Bertsimas and Sim, 2004, for details). A robust solution for a given $\Gamma$ value can still provide adequate protection even when the system disruption exceeds the budget (see Leus and Herroelen, 2007, for example).

Consider the nonlinear formulation in which constraints $1 \mathrm{~b}$ are replaced by the following expression, which states that each machine should still respect the deadline even if at most $\Gamma$ jobs are disrupted:

$$
\sum_{j \in J} \bar{p}_{j} x_{i j}+\max _{S \subset J:|S| \leq \Gamma} \sum_{j \in S} \hat{p}_{j} x_{i j} \leq T y_{i} \quad \forall i \in M
$$

For a given solution vector $\boldsymbol{x}^{*}$, for machine $i$, the protection associated with the subproblem in (2) can be defined as $\beta_{i}\left(\boldsymbol{x}^{*}, \Gamma\right)=\max _{S \subset J|| S \mid \leq \Gamma} \sum_{j \in S} \hat{p}_{j} x_{i j}^{*}$, which can be obtained through the following 
linear model:

$$
\begin{array}{lll}
\beta_{i}\left(\boldsymbol{x}^{*}, \Gamma\right)=\operatorname{maximize} & \sum_{j \in J} \hat{p}_{j} x_{i j}^{*} z_{i j} & \\
\text { subject to } & \sum_{j \in J} z_{i j} \leq \Gamma & \\
& 0 \leq z_{i j} \leq 1 \quad \forall j \in J
\end{array}
$$

The solution of $\beta_{i}\left(x^{*}, \Gamma\right)$ is equivalent to sorting the jobs assigned to machine $i$ in non-increasing order of their deviations and picking at most $\Gamma$ of them starting from the head of the list. In formulation (3) we associate the dual variables $s_{i}$ with constraints (3b), and dual variables $q_{i j}$ with (3c). The dual of formulation (3) is then:

$$
\begin{array}{rlr}
\operatorname{minimize} & \Gamma s_{i}+\sum_{j \in J} q_{i j} & \\
\text { subject to } & s_{i}+q_{i j} \geq \hat{p}_{j} x_{i j}^{*} & \forall j \in J \\
& q_{i j} \geq 0 & \forall j \in J \\
& s_{i} \geq 0 &
\end{array}
$$

From strong duality, we know that the optimal objective value of problem (4) equals $\beta_{i}\left(\boldsymbol{x}^{*}, \Gamma\right)$. Substituting (4) into (2), the robust counterpart RMAP of the machine availability problem can 
then be captured by the following linear formulation:

$$
\begin{array}{rrr}
\operatorname{minimize} & \sum_{i \in M} y_{i} & \\
\text { subject to } & \sum_{j \in J} \bar{p}_{j} x_{i j}+\Gamma s_{i}+\sum_{j \in J} q_{i j} \leq T y_{i} & \forall i \in M \\
& s_{i}+q_{i j} \geq \hat{p}_{j} x_{i j} & \\
& \sum_{i \in M} x_{i j}=1 & \forall i \in M, \forall j \in J \\
& x_{i j} \in\{0,1\} & \forall j \in J \\
& y_{i} \in\{0,1\} & \\
& q_{i j} \geq 0 & \forall i \in M \\
& s_{i} \geq 0 & \forall j \in J \\
& \forall i \in M, \forall j \in J
\end{array}
$$

\section{Branch and price}

It is well known that the intuitive formulation (1) produces a weak LP bound and suffers from inherent symmetry, and is thus not efficiently solvable in practice (Valério De Carvalho, 2002). It can be inferred that the computational behavior of the robust counterpart (5) would be poor as well. There have been some attempts to improve the performance of the intuitive model by adding valid inequalities and symmetry-breaking inequalities, which are surveyed in Delorme et al. (2016). Despite those efforts, the computational performance of the intuitive formulation and its robust counterpart remain unsatisfying. For a better computational performance, a set covering formulation for RMAP is presented in this section, followed by the associated pricing problem. These two components form the basis of a $\mathrm{B} \& \mathrm{P}$ procedure. Pseudo-polynomial formulations for the BPP have also been intensively studied (see, for example, Valério De Carvalho, 2002; Dyckhoff, 1981), and have proved to be very effective (Delorme et al., 2016). Due to the uncertain "item sizes" in RMAP, however, these formulations cannot be easily applied. 


\subsection{Set covering formulation}

Let $G$ denote the set of all feasible job groups that can be executed on the same machine within the time limit. A binary variable $\lambda_{g}$ for each job group $g \in G$ is introduced so that $\lambda_{g}$ takes value 1 if the group is chosen and 0 otherwise. The goal is to choose the minimum number of job groups such that each job is contained in one group. The set covering formulation is then:

$$
\begin{array}{rlr}
\operatorname{minimize} & \sum_{g \in G} \lambda_{g} & \\
\text { subject to } & \sum_{g \in G: j \in g} \lambda_{g} \geq 1 & \forall j \in J \\
& \lambda_{g} \in\{0,1\} & \forall g \in G
\end{array}
$$

The objective function (6a) is to minimize the number of selected job groups. The constraints $6 \mathrm{~b}$ indicate that each job should be carried out on a machine. It would be intuitive to state the constraints $6 \mathrm{~b}$ as equalities, leading to a partitioning formulation, but the above format yields a better performance for solving the LP relaxation because its dual variables are more constrained. Thereby, the LP relaxation of the set covering formulation is usually more stable and converges faster (Lopes and Valério De Carvalho, 2007).

The set covering formulation has an exponential number of decision variables, so directly generating all these variables is not practical and we develop a B\&P procedure, using CG Gilmore and Gomory, 1961) to solve the LP relaxation at each node of the B\&P tree. Similar B\&P and CG procedures have been extensively investigated for parallel machine scheduling with various objectives (e.g., Chen and Powell, 1999 ; van den Akker et al., 1999). 


\subsection{The pricing problem}

For the LP relaxation of model (6) we remove the integrality constraints and the upper bound $\lambda_{g} \leq 1$. With dual variables $\pi_{j}$ associated to constraints $6 \mathrm{~b}$, we obtain the following dual:

$$
\begin{array}{rlr}
\operatorname{maximize} & \sum_{j \in J} \pi_{j} & \\
\text { subject to } & \sum_{j \in g} \pi_{j} \leq 1 & \forall g \in G \\
& \pi_{j} \geq 0 & \forall j \in J
\end{array}
$$

At each CG iteration it is checked whether there exist violated constraints in $(7 \mathrm{~b})$. If not then the optimal solution of the full master (6) is obtained; otherwise a new column is generated to improve the current solution.

Given the current dual solution $\pi^{*}$, the pricing problem is to find out whether there exists a job group $g \in G$ with $\sum_{j \in g} \pi_{j}^{*}>1$. By identifying constraints that are violated most in each iteration, we generate new columns with the lowest reduced cost. The pricing problem is then:

$$
\begin{aligned}
\operatorname{maximize} & \sum_{j \in J} \pi_{j}^{*} a_{j} \\
\text { subject to } & \sum_{j \in J} \bar{p}_{j} a_{j}+\max _{S \subset J:|S| \leq \Gamma} \sum_{j \in S} \hat{p}_{j} a_{j} \leq T \\
& a_{j} \in\{0,1\} \quad \forall j \in J
\end{aligned}
$$

Model (8) is essentially a robust variant of the knapsack problem, and with a similar linearization process as before, model (8) is equivalent to the following model, where $\theta$ and $\xi_{j}$ are the dual variables in the reformulation of the subproblem in constraint $8 \mathrm{~b}$ ). 


$$
\begin{array}{cll}
\operatorname{maximize} & \sum_{j \in J} \pi_{j}^{*} a_{j} & \\
\text { subject to } & \sum_{j \in J} \bar{p}_{j} a_{j}+\Gamma \theta+\sum_{j \in J} \xi_{j} \leq T & \\
& \theta+\xi_{j} \geq \hat{p}_{j} a_{j} & \forall j \in J \\
& a_{j} \in\{0,1\} & \forall j \in J \\
& \xi_{j} \geq 0 & \forall j \in J \\
& \theta \geq 0 &
\end{array}
$$

\subsection{Bounding procedure for column generation}

It is generally not necessary to solve the master problem to optimality to obtain a lower bound for the IP solution $z_{I P}$. A valid lower bound on $z_{I P}$ can be given based on the optimal subproblem

solution following Farley (1990). In a given B\&P node $u$, this bound is $\left\lceil z_{L P}^{u} / v^{u}\right\rceil$, where $z_{L P}^{u}$ is the incumbent optimal LP solution for the restricted master and $v^{u}$ is the objective value of the pricing problem. This bound has been shown to be effective for the CSP and the BPP (Vanderbeck, 1999), and it has already been widely used in B\&P procedures for the one-dimensional CSP (see Vance, 1998, and Vanderbeck, 2000). In our implementation, the CG process for the root node $r$ in the $\mathrm{B} \& \mathrm{P}$ tree is terminated when $\left\lceil z_{L P}^{r}\right\rceil=\left\lceil z_{L P}^{r} / v^{r}\right\rceil$ holds. Given $z_{L B}=\left\lceil z_{L P}^{r}\right\rceil$, at subsequent nodes in the $\mathrm{B} \& \mathrm{~B}$ tree, once the LP relaxation is below this bound then nothing is gained by solving the LP to optimality. Therefore, in the following nodes of the B\&P tree, whenever the LP solution objective is below $z_{L B}$, CG stops and branching is carried out.

\subsection{The branching rule}

For the BPP and the CSP, different branching schemes have been proposed. Vanderbeck (1999) branches on a set of generated columns with a fractional sum in the LP solution, while Belov and Scheithauer (2006) branch on individual fractional variables. In this paper we apply the more generic Ryan and Foster branching rule. The idea is to identify two jobs $j, j^{\prime} \in J$ such that $0<\sum_{g: j, j^{\prime} \in g} \lambda_{g}<1$, which are then enforced to be either both assigned to the same machine, or 
to two different machines. Upon branching, two child nodes are generated for a parent node. In the first child node, columns with $a_{j} \neq a_{j^{\prime}}$ are removed from the master and constraint $a_{j}=a_{j^{\prime}}$ is added to the subproblem. In the second child, the new constraint $a_{j}+a_{j^{\prime}} \leq 1$ is included in the pricing problem and columns with $a_{j}+a_{j^{\prime}}=2$ are removed from the master column pool.

In the root node, one may opt for DP to solve the pricing problem. With all jobs sorted in non-increasing order of their processing-time deviations, a DP procedure can use the value function $V(j, t, c)$, representing the objective value for the first $j$ jobs within time $t$ and with $c$ disrupted jobs. The resulting DP recursion has time and space complexity $O(n T \Gamma)$. The advantage of the Ryan and Foster branching rule is that it preserves the structure of the master problem. Introducing branching constraints, however, changes the structure of the pricing problem for the non-root nodes of the B\&P tree, and the foregoing DP procedure can no longer be applied. Without robustness considerations, one could merge jobs $j$ and $j^{\prime}$ for imposing constraint $a_{j}=a_{j^{\prime}}$, while the requirement $a_{j}+a_{j^{\prime}} \leq 1$ would imply that the two jobs are not allowed on the same machine. The pricing problem is then essentially a robust variant of the knapsack problem with conflicts (see Pferschy and Schauer, 2009). Keeping track of the (conflict) graph structure and providing a feasible independent set with the largest weight would already impose great difficulties upon a traditional DP procedure. When processing times can fluctuate, however, as is the case in this paper, simply merging two jobs is no longer possible. Consequently, the pricing problem becomes quite intractable in this B\&P procedure. It is shown in Section 6 that solving formulation (9) with a MIP solver cannot guarantee a consistently good performance. We therefore resort to ZDDs for solving the pricing problem.

\section{$5 \quad$ Pricing with ZDDs}

\subsection{ZDDs}

Binary decision diagrams (BDDs) were originally proposed by Lee (1959) and Akers (1978). A BDD is a directed acyclic graph that can be seen as a reduced binary decision tree. With BDDs, a Boolean function with a set of binary variables can be compactly and uniquely represented. Later on, ZDDs were introduced by Minato (1993) as extensions of BDDs with new reduction rules. Both BDDs and ZDDs can be used to represent and manipulate sets of combinations. 


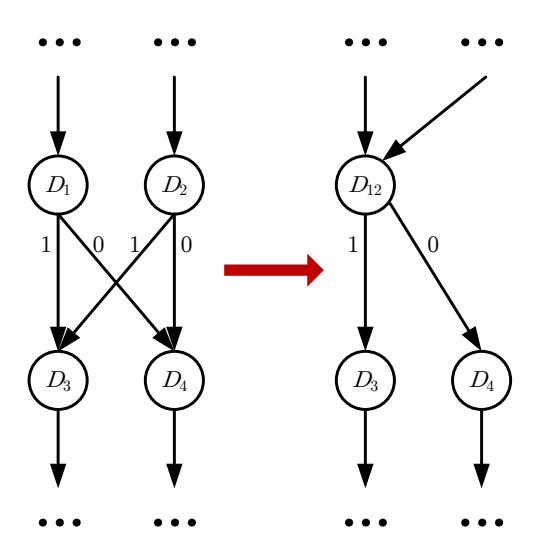

(a) Node merge in both BDDs and ZDDs

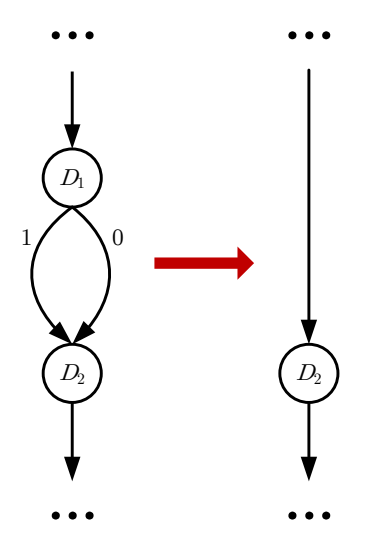

(b) Node deletion in BDDs

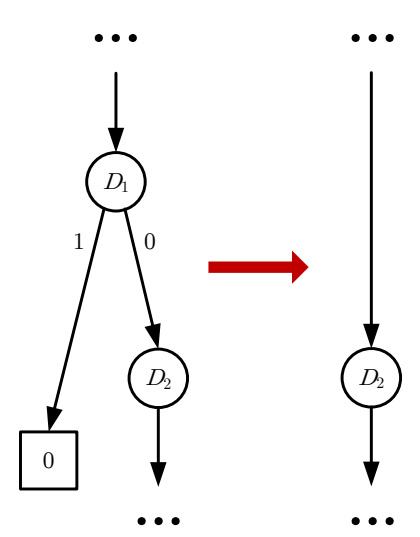

(c) Node deletion in ZDDs

Figure 1: The reduction rules for BDDs and ZDDs

A ZDD $Z$ has one root node, and two terminal nodes 0-terminal and 1-terminal. Every path in $Z$ ending at the 1-terminal node represents a valid combination. Each non-terminal node at one specific level in $Z$ is labeled by the corresponding stage in the decision process. Each non-terminal node has two outgoing edges, the 0 -edge and the 1-edge, which point to the two child nodes 0-child and 1-child, respectively. Uncompressed decision diagrams (DDs) can be reduced by merging nodes at the same level that have the same successor nodes, as shown in Figure 1(a). Both BDDs and ZDDs use this reduction rule. The difference is that BDDs use a node deletion rule to eliminate redundant nodes whose two edges point to the same node (see Figure 1(b)), while ZDDs delete nodes whose 1-edge points to the 0-terminal node and connect their incoming edges directly to the node reached by its 0-edge (as in Figure 1(c)). These reduction rules can be applied recursively in the decision diagrams from bottom to top. Compared with BDDs, ZDDs have the merit that each path from the root to the terminal nodes represents a unique combination. Thus, ZDDs are more suitable for representing sets of combinations than the original BDDs (Minato, 2001).

To the best of our knowledge, not so much work has been done on utilizing decision diagrams for solving combinatorial optimization problems. Hooker (2013) shows the relationship between the state transition graph of a DP procedure and a decision diagram, revealing the possibility of solving problems amenable to DP with decision diagrams. Bergman et al. (2016) propose a general $\mathrm{B} \& \mathrm{~B}$ algorithm for discrete optimization with a BDD-based solver instead of LP relaxation to produce tighter bounds. Morrison et al. (2016) first use ZDDs in solving pricing problems in a B\&P algorithm for the graph coloring problem. Kowalczyk and Leus (2018) study parallel machine 
scheduling to minimize the sum of weighted completion times, where ZDDs are utilized for solving the pricing problem. These works show the potential of ZDDs to compactly represent and search the column pool for a pricing problem.

\subsection{Construction of the ZDDs}

Building a ZDD $Z_{\mathcal{F}}$ representing a family $\mathcal{F}$ of subsets of the job set $J$ can be achieved in different ways. Knuth (2009) proposes a recursive procedure that constructs a ZDD representing a set of paths between two nodes in an undirected graph. Iwashita and Minato (2013) introduce a top-down construction framework for constructing a ZDD with recursive specification for a family of subsets; their framework is adopted and customized in this paper.

A configuration is a pair $\langle j, s\rangle$, which is used to label a node in a ZDD, where $j \in\{1, \ldots, n\}$ is the node level corresponding to a job index and $s$ is the state of that node containing key information. The state $s$ is a tuple $\langle t, c\rangle$, where the former is the cumulative time and the latter is the worst-case job counter. Pre-defined configurations $\langle n+1,0\rangle$ and $\langle n+1,1\rangle$ are associated to the 0-terminal node and 1-terminal node respectively. The top-down construction framework relies on the recursive specification, which internally guides the construction process. A recursive specification $S$ of a ZDD is defined by a pair of functions:

- $R O O T()$ : this function takes no argument and returns the configuration of the root node;

- $C H I L D(\langle j, s\rangle, b)$ : this function takes the configuration $\langle j, s\rangle$ of a node and a binary number $b \in\{0,1\}$ as arguments, and returns the configuration for the b-child of that node.

The recursive specification gives the blueprint of a ZDD as it describes the structure of the ZDD in an implicit but compact way. With the recursive specification provided, the top-down construction procedure for a ZDD is shown in Algorithm 1. It first constructs a DD in a breadthfirst manner based on the given recursive specification. Then it compresses the DD structure using the reduction rules for ZDDs. It should also be pointed out that before building the ZDD, the jobs should be sorted in non-increasing order of the processing-time deviation. In this paper, in order to use the top-down construction framework, the recursive specification is specialized and redesigned. The recursive specification for finding all reachable configurations in the construction process is given in Algorithm 2 . 


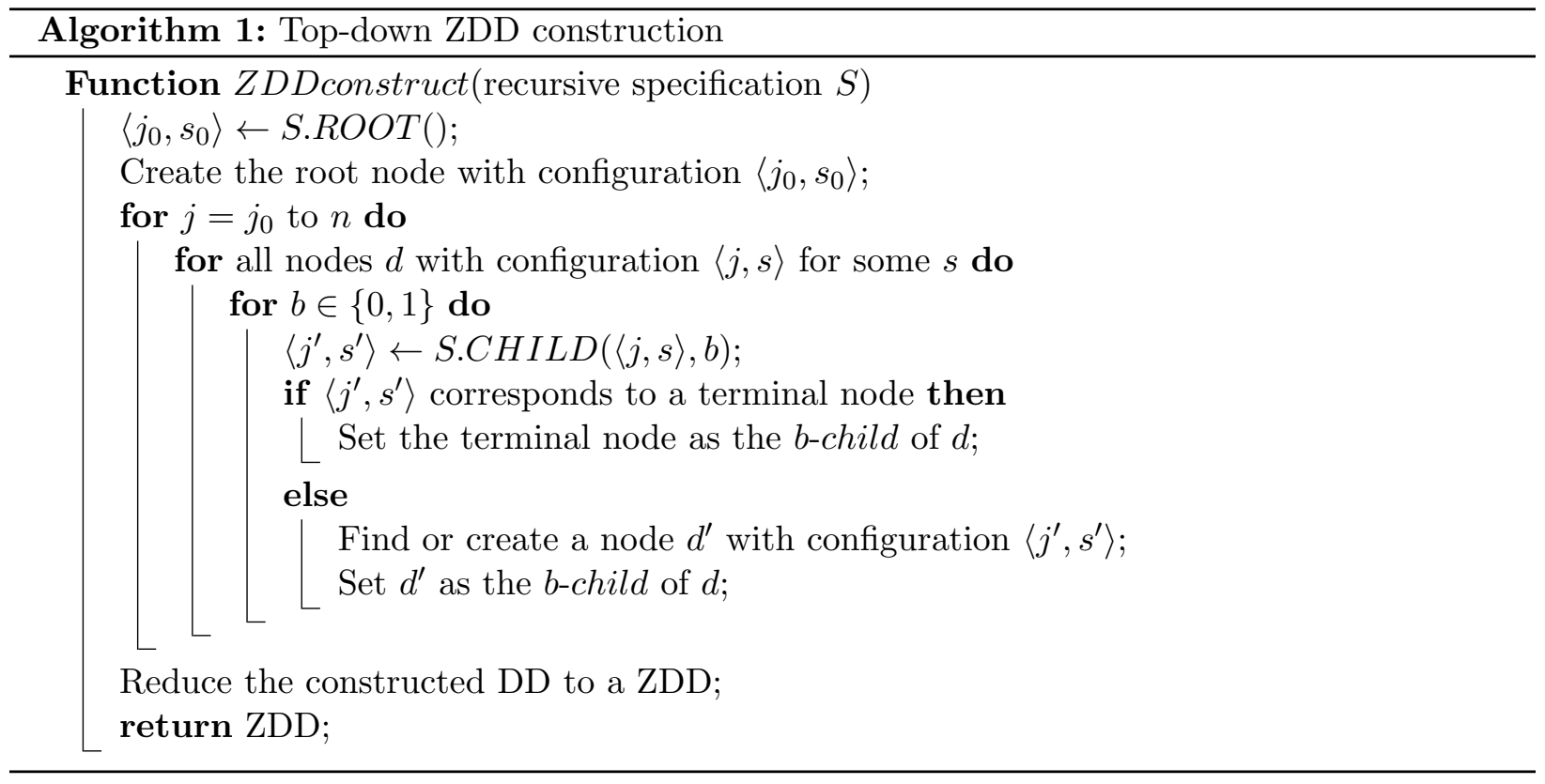

Figure 2 illustrates the construction process for a ZDD on a four-job example instance with $\Gamma=2$ and $T=16$. Detailed data for the example instance are given in Table 1. In Figure 2, the levels in the ZDD are indicated on the left, and the state of each node is shown in the label. All 1-edges are drawn in solid lines while the 0-edges appear as dotted lines. In Figure 2(a) the DD is initialized and the 1-child and 0-child at level 2 are identified. In Figure 2(b), all the children of the nodes at level 2 are then configured. It can be seen that the 1-edge of $d_{2}$ skips level 3 . This is because the 1-edge of $d_{2}$ to level 3 leads to the 1-edge of its 1-child ending up with the 0 -terminal, as job set $\{1,2,3\}$ is not a feasible job group. Thus $d_{2}$ 's 1 -child at level 3 is eliminated automatically; this directly follows the reduction rule of ZDDs. In Figure 2(c), the children of the nodes at level 3 are determined, and then all feasible paths are made to terminate in the 1-terminal in Figure 2(d). After the construction of the DD, the reduction process is applied and the ZDD in Figure 2(e) is obtained. The reduction process is a bottom-up recursion. All nodes at level 4 in Figure 2(d) share the same child (1-terminal) and are merged as node $Z_{5}$ in Figure 2(e), afterwards all the nodes at level 3 of Figure 2(d) can also be merged as node $Z_{4}$ in Figure 2(e). Each path from the root to the 1-terminal in the ZDD represents a feasible solution for the pricing problem, so once the ZDD for a node in the B\&P tree is created we simply identify a longest path with the dual prices from the master as the lengths for the 1-edges. In this example the optimal objective value is 7 , achieved by the job group $\{1,2,4\}$. 


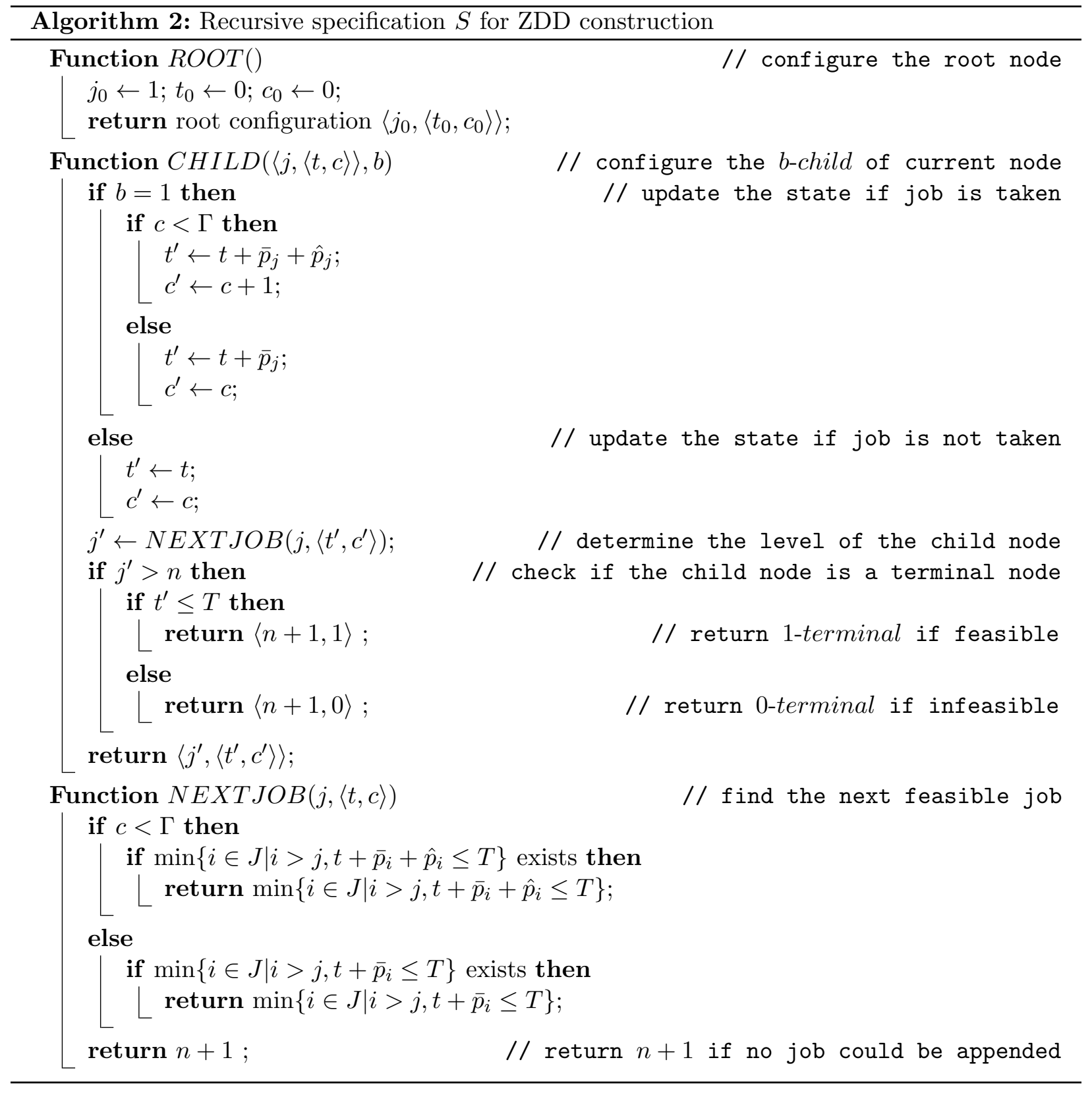

\subsection{Branching with ZDDs}

Upon branching in the $\mathrm{B} \& \mathrm{P}$ tree, new constraints are to be imposed in the pricing problems following the Ryan and Foster branching rule. The branching constraints need to be reflected in the ZDD structure of the child nodes; this can be efficiently achieved by using the generic intersection operation for ZDDs. Figure 3 illustrates this process. Figure 3(a) contains the ZDD in the root node for the example instance, which contains all feasible solutions. We illustrate branching on jobs 1 and 3. Figure 3(b) depicts the ZDD containing all job combinations with only constraint $a_{1}=a_{3}$ 
Table 1: A four-job instance with $\Gamma=2$ and $T=16$.

\begin{tabular}{cccc}
\hline job $j$ & $\bar{p}_{j}$ & $\hat{p}_{j}$ & $\pi_{j}^{*}$ \\
\hline 1 & 4 & 2 & 3 \\
2 & 5 & 2 & 3 \\
3 & 4 & 2 & 2 \\
4 & 3 & 1 & 1 \\
\hline
\end{tabular}

imposed. Similarly, the ZDD in Figure 3(c) represents the set of all job sets satisfying $a_{1}+a_{3} \leq 1$. By making the intersection of Figure 3(a) and Figure 3(b), the new ZDD with constraint $a_{1}=a_{3}$ is then obtained in Figure 3(d). The intersection of Figure 3(a) and Figure 3(c) represents all feasible job groups with $a_{1}+a_{3} \leq 1$, which is given in Figure 3(e).

In our experiments, we adopt the implementation choices of Kowalczyk and Leus (2018) to impose the branching constraints to the ZDDs of the non-root nodes in the $\mathrm{B} \& \mathrm{P}$ tree. In order to avoid drastic changes to the structure of ZDDs and to keep the number of nodes in the ZDDs manageable, job pairs with close job indices are preferably chosen for branching: among all job pairs that can be branched on after acquiring the non-integral LP solution, a pair $j, j^{\prime}$ with the smallest difference $\left|j-j^{\prime}\right|$ is selected.

\section{Computational experiments}

\subsection{Experimental setup}

All algorithms are implemented in Visual $\mathrm{C}++$. The computational experiments are performed on a PC equipped with Intel Core i7-4790 CPU at $3.6 \mathrm{GHz}$ with $8 \mathrm{~GB}$ of RAM on a Windows 10 64-bit OS. All LPs and MIPs are solved with CPLEX 12.6.3 using Concert Technology with default settings. The ZDD-based pricing solver uses one thread on one core. For the MIP pricing model and other MIP models implemented with CPLEX, the query callback is incorporated in order to terminate the solving process when it exceeds the time limit, thus only one thread is used as well (see the user's manual of CPLEX IBM, 2015). The algorithms are tested on a diverse set of instances, which are generated randomly as follows. The number of jobs $n$ is between 30 and 180 (below also referred to as instance size). The processing times are integers uniformly drawn from interval $[1,20]$ or $[1,100]$ for most instances (below referred to as p-range). The processing-time deviation is 0.2 times the processing time, rounded to the nearest higher integer. Five robustness 


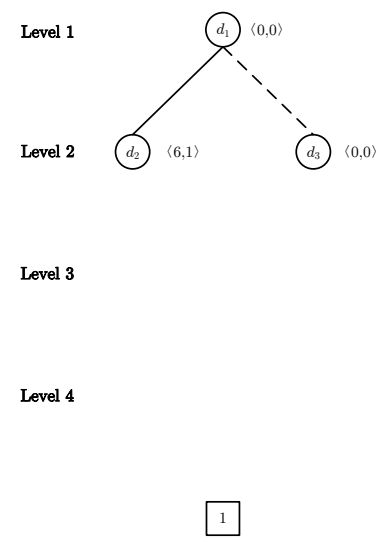

(a)

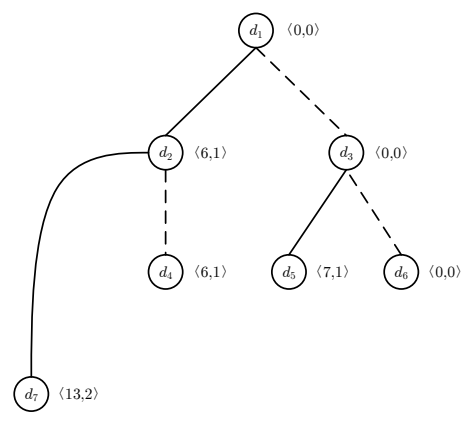

(b)

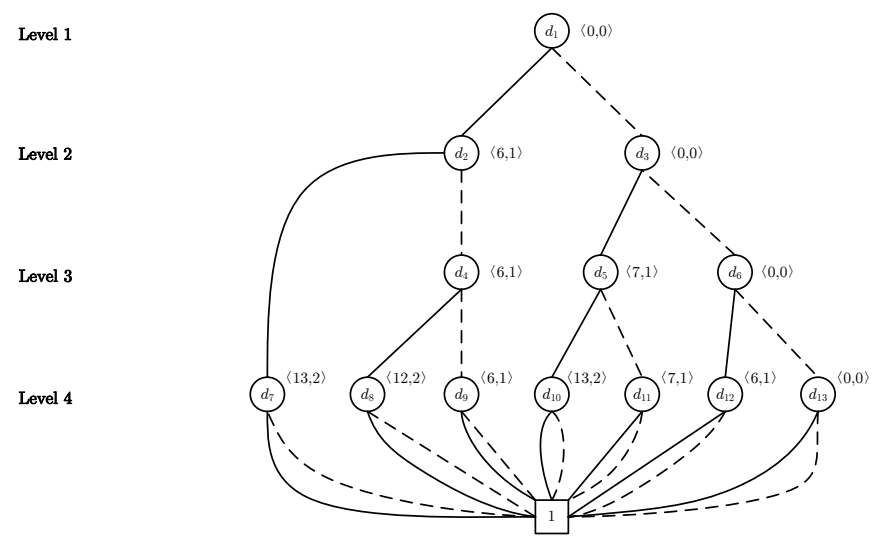

(d)

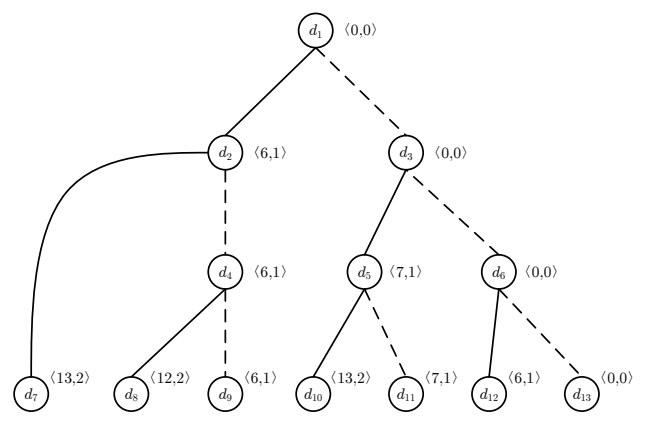

(c)

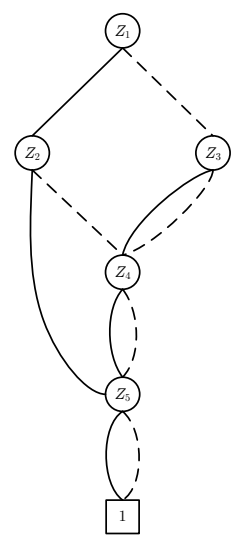

(e)

Figure 2: The ZDD construction process for the example instance

levels are considered by varying $\Gamma=\lceil\gamma n\rceil$ with $\gamma$ either $0 \%, 5 \%, 10 \%, 15 \%$ or $20 \%$. Four deadlines are generated for each instance, which are equal to a fraction frac of the sum of the worst-case job processing times; we examine frac $=1 / 4,1 / 6,1 / 8$ and 1/10. For each combination of parameter settings, 10 instances are randomly generated. All instances tested are available online at the webpage http://feb.kuleuven.be/roel.leus/public/RMAP-instances.htm. The time limit for each run of the algorithms on one instance is 1200 seconds (20 minutes).

\subsection{Computational results}

In the tables, the entries for opt show the number of instances solved to optimality within the time limit, out of 10 instances per setting. Columns labeled by time contain the average CPU time in seconds. We first show the results of the intuitive formulation (5) for instances of size 30 in Table 2. Clearly, the intuitive formulation already struggles with these small instances, especially 
Table 2: Computational results of the intuitive formulation on instances with $n=30$

\begin{tabular}{|c|c|c|c|c|c|}
\hline \multirow[b]{2}{*}{$\gamma$} & \multirow[b]{2}{*}{ frac } & \multicolumn{2}{|c|}{$p$-range $[1,20]$} & \multicolumn{2}{|c|}{ p-range $[1,100]$} \\
\hline & & $o p t$ & time & $o p t$ & time \\
\hline \multirow[t]{4}{*}{$0 \%$} & $1 / 4$ & 10 & 0.07 & 10 & 0.06 \\
\hline & $1 / 6$ & 10 & 0.07 & 10 & 0.08 \\
\hline & $1 / 8$ & 10 & 0.06 & 10 & 0.06 \\
\hline & $1 / 10$ & 10 & 0.06 & 10 & 0.06 \\
\hline \multirow[t]{4}{*}{$5 \%$} & $1 / 4$ & 10 & 0.28 & 10 & 0.28 \\
\hline & $1 / 6$ & 10 & 0.45 & 10 & 0.40 \\
\hline & $1 / 8$ & 10 & 1.39 & 10 & 1.16 \\
\hline & $1 / 10$ & 10 & 122.63 & 10 & 4.70 \\
\hline \multirow[t]{4}{*}{$10 \%$} & $1 / 4$ & 10 & 0.32 & 10 & 0.33 \\
\hline & $1 / 6$ & 10 & 0.45 & 10 & 0.76 \\
\hline & $1 / 8$ & 10 & 1.58 & 10 & 2.36 \\
\hline & $1 / 10$ & 4 & 723.38 & 4 & 751.91 \\
\hline \multirow[t]{4}{*}{$15 \%$} & $1 / 4$ & 10 & 0.40 & 10 & 0.58 \\
\hline & $1 / 6$ & 10 & 1.20 & 10 & 2.50 \\
\hline & $1 / 8$ & 3 & 845.62 & 5 & 676.35 \\
\hline & $1 / 10$ & 4 & 859.89 & 0 & 1200.00 \\
\hline \multirow[t]{4}{*}{$20 \%$} & $1 / 4$ & 10 & 0.65 & 10 & 0.80 \\
\hline & $1 / 6$ & 10 & 1.34 & 10 & 128.75 \\
\hline & $1 / 8$ & 2 & 1002.89 & 2 & 986.60 \\
\hline & $1 / 10$ & 2 & 962.08 & 0 & 1200.00 \\
\hline \multicolumn{2}{|c|}{ Overall } & 165 & 226.24 & 161 & 247.89 \\
\hline
\end{tabular}

Table 3: Computational results of the two B\&P algorithms on instances with $n=60$

\begin{tabular}{|c|c|c|c|c|c|c|c|c|c|c|c|c|c|}
\hline \multirow[b]{3}{*}{$\gamma$} & \multirow[b]{3}{*}{ frac } & \multicolumn{6}{|c|}{ BP-ZDD } & \multicolumn{6}{|c|}{ BP-MIP } \\
\hline & & \multicolumn{3}{|c|}{$p$-range $[1,20]$} & \multicolumn{3}{|c|}{$p$-range $[1,100]$} & \multicolumn{3}{|c|}{$p$-range $[1,20]$} & \multicolumn{3}{|c|}{$p$-range $[1,100]$} \\
\hline & & $o p t$ & time & pricing & opt & time & pricing & opt & time & pricing & $o p t$ & time & pricing \\
\hline \multirow[t]{4}{*}{$0 \%$} & $1 / 4$ & 10 & 6.54 & 1.39 & 10 & 9.31 & 4.04 & 10 & 41.41 & 37.65 & 10 & 44.31 & 40.23 \\
\hline & $1 / 6$ & 10 & 7.28 & 1.19 & 10 & 11.65 & 3.37 & 10 & 79.88 & 74.44 & 10 & 153.87 & 145.50 \\
\hline & $1 / 8$ & 10 & 5.17 & 1.00 & 10 & 6.52 & 2.35 & 10 & 46.88 & 43.50 & 10 & 59.72 & 55.83 \\
\hline & $1 / 10$ & 10 & 3.47 & 0.69 & 10 & 5.61 & 2.07 & 10 & 36.46 & 33.75 & 10 & 42.70 & 39.89 \\
\hline \multirow[t]{4}{*}{$5 \%$} & $1 / 4$ & 10 & 5.87 & 1.38 & 10 & 8.80 & 3.83 & 10 & 59.81 & 55.60 & 10 & 72.63 & 67.83 \\
\hline & $1 / 6$ & 10 & 4.43 & 1.06 & 10 & 7.12 & 3.12 & 10 & 52.95 & 49.66 & 10 & 59.90 & 56.43 \\
\hline & $1 / 8$ & 10 & 4.25 & 0.92 & 10 & 5.79 & 2.30 & 10 & 66.42 & 62.85 & 10 & 64.56 & 61.36 \\
\hline & $1 / 10$ & 10 & 3.65 & 0.68 & 10 & 4.67 & 1.59 & 10 & 58.08 & 55.26 & 10 & 59.44 & 56.52 \\
\hline \multirow[t]{4}{*}{$10 \%$} & $1 / 4$ & 10 & 6.17 & 1.38 & 10 & 10.50 & 4.76 & 10 & 70.77 & 66.17 & 10 & 87.10 & 81.76 \\
\hline & $1 / 6$ & 10 & 5.15 & 1.17 & 10 & 8.96 & 3.74 & 10 & 80.11 & 75.80 & 10 & 101.97 & 96.80 \\
\hline & $1 / 8$ & 10 & 5.98 & 1.12 & 10 & 8.01 & 2.61 & 10 & 99.94 & 95.36 & 10 & 121.62 & 116.46 \\
\hline & $1 / 10$ & 10 & 5.16 & 0.72 & 10 & 6.60 & 1.84 & 10 & 209.17 & 204.45 & 10 & 153.30 & 148.91 \\
\hline \multirow[t]{4}{*}{$15 \%$} & $1 / 4$ & 10 & 6.70 & 1.56 & 10 & 12.93 & 6.27 & 10 & 86.21 & 81.45 & 10 & 111.72 & 105.55 \\
\hline & $1 / 6$ & 10 & 7.37 & 1.55 & 10 & 12.66 & 5.18 & 10 & 145.99 & 139.62 & 10 & 226.34 & 219.67 \\
\hline & $1 / 8$ & 10 & 7.35 & 1.12 & 10 & 10.20 & 3.39 & 6 & 664.41 & 660.80 & 5 & 828.69 & 825.43 \\
\hline & $1 / 10$ & 10 & 4.72 & 0.68 & 10 & 7.50 & 1.89 & 4 & 866.02 & 864.19 & 4 & 1057.86 & 1055.02 \\
\hline \multirow[t]{4}{*}{$20 \%$} & $1 / 4$ & 10 & 7.83 & 2.08 & 10 & 17.53 & 8.99 & 10 & 117.26 & 111.28 & 10 & 154.98 & 148.07 \\
\hline & $1 / 6$ & 10 & 8.31 & 1.51 & 10 & 14.23 & 5.82 & 5 & 865.83 & 862.93 & 3 & 1150.78 & 1148.33 \\
\hline & $1 / 8$ & 10 & 7.19 & 1.01 & 10 & 10.18 & 3.13 & 3 & 991.74 & 989.95 & 0 & 1200.00 & 1199.12 \\
\hline & $1 / 10$ & 10 & 4.09 & 0.69 & 10 & 6.63 & 1.56 & 1 & 1191.50 & 1190.38 & 0 & 1200.00 & 1199.66 \\
\hline \multicolumn{2}{|c|}{ Overall } & 200 & 5.83 & 1.15 & 200 & 9.27 & 3.59 & 169 & 291.54 & 287.75 & 162 & 347.57 & 343.42 \\
\hline
\end{tabular}


Table 4: Computational results of the two B\&P algorithms on instances with $n=90$

\begin{tabular}{|c|c|c|c|c|c|c|c|c|c|c|c|c|c|}
\hline \multirow[b]{3}{*}{$\gamma$} & \multirow[b]{3}{*}{ frac } & \multicolumn{6}{|c|}{ BP-ZDD } & \multicolumn{6}{|c|}{ BP-MIP } \\
\hline & & \multicolumn{3}{|c|}{$p$-range $[1,20]$} & \multicolumn{3}{|c|}{$p$-range $[1,100]$} & \multicolumn{3}{|c|}{$p$-range $[1,20]$} & \multicolumn{3}{|c|}{$p$-range $[1,100]$} \\
\hline & & $o p t$ & time & pricing & $o p t$ & time & pricing & $o p t$ & time & pricing & $o p t$ & time & pricing \\
\hline \multirow[t]{4}{*}{$0 \%$} & $1 / 4$ & 10 & 20.83 & 7.13 & 10 & 35.13 & 22.50 & 10 & 123.40 & 112.37 & 10 & 114.83 & 103.22 \\
\hline & $1 / 6$ & 10 & 20.12 & 6.77 & 10 & 48.90 & 25.23 & 10 & 148.51 & 138.93 & 10 & 325.69 & 305.53 \\
\hline & $1 / 8$ & 10 & 14.16 & 5.60 & 10 & 29.23 & 18.73 & 10 & 98.06 & 90.79 & 10 & 111.48 & 103.51 \\
\hline & $1 / 10$ & 10 & 11.87 & 4.71 & 10 & 23.19 & 14.72 & 10 & 77.27 & 71.64 & 10 & 90.76 & 84.24 \\
\hline \multirow[t]{4}{*}{$5 \%$} & $1 / 4$ & 10 & 21.48 & 7.78 & 10 & 42.11 & 27.28 & 10 & 158.04 & 146.18 & 10 & 165.15 & 153.06 \\
\hline & $1 / 6$ & 10 & 16.15 & 7.34 & 10 & 32.83 & 23.63 & 10 & 119.95 & 112.67 & 10 & 129.59 & 121.91 \\
\hline & $1 / 8$ & 10 & 14.42 & 6.11 & 10 & 28.50 & 19.16 & 10 & 178.53 & 171.71 & 10 & 166.89 & 159.15 \\
\hline & $1 / 10$ & 10 & 13.46 & 5.22 & 10 & 23.26 & 14.29 & 10 & 283.37 & 276.72 & 10 & 204.43 & 196.89 \\
\hline \multirow[t]{4}{*}{$10 \%$} & $1 / 4$ & 10 & 25.61 & 10.57 & 10 & 43.37 & 29.64 & 10 & 175.81 & 163.43 & 10 & 180.42 & 168.65 \\
\hline & $1 / 6$ & 10 & 20.99 & 10.86 & 10 & 47.82 & 34.79 & 10 & 191.85 & 182.80 & 10 & 225.12 & 214.88 \\
\hline & $1 / 8$ & 10 & 24.81 & 11.06 & 10 & 45.45 & 30.51 & 10 & 314.02 & 302.17 & 9 & 418.58 & 408.08 \\
\hline & $1 / 10$ & 10 & 25.06 & 9.41 & 10 & 40.32 & 23.47 & 8 & 619.60 & 608.35 & 5 & 912.36 & 904.10 \\
\hline \multirow[t]{4}{*}{$15 \%$} & $1 / 4$ & 10 & 31.52 & 15.87 & 10 & 73.95 & 56.08 & 9 & 314.41 & 302.12 & 10 & 293.92 & 277.05 \\
\hline & $1 / 6$ & 10 & 41.28 & 21.00 & 10 & 81.78 & 57.57 & 10 & 395.27 & 379.04 & 4 & 1028.83 & 1021.92 \\
\hline & $1 / 8$ & 10 & 37.84 & 15.31 & 10 & 54.95 & 34.84 & 1 & 1178.22 & 1176.47 & 0 & 1200.00 & 1199.53 \\
\hline & $1 / 10$ & 10 & 31.69 & 12.57 & 10 & 44.07 & 27.09 & 0 & 1200.00 & 1199.62 & 0 & 1200.00 & 1199.51 \\
\hline \multirow[t]{4}{*}{$20 \%$} & $1 / 4$ & 10 & 43.25 & 26.85 & 10 & 106.13 & 86.10 & 10 & 284.13 & 268.64 & 10 & 394.19 & 375.31 \\
\hline & $1 / 6$ & 10 & 49.19 & 26.20 & 10 & 85.89 & 62.54 & 0 & 1200.00 & 1199.64 & 0 & 1200.00 & 1199.58 \\
\hline & $1 / 8$ & 10 & 39.46 & 19.04 & 10 & 64.05 & 41.84 & 0 & 1200.00 & 1199.73 & 0 & 1200.00 & 1199.64 \\
\hline & $1 / 10$ & 10 & 36.05 & 18.50 & 10 & 53.00 & 33.92 & 0 & 1200.00 & 1199.67 & 0 & 1200.00 & 1199.66 \\
\hline \multicolumn{2}{|c|}{ Overall } & 200 & 26.96 & 12.40 & 200 & 50.20 & 34.20 & 148 & 473.02 & 465.13 & 138 & 538.11 & 529.77 \\
\hline
\end{tabular}

Table 5: Computational results of the two B\&P algorithms on instances with $n=120$

\begin{tabular}{|c|c|c|c|c|c|c|c|c|c|c|c|c|c|}
\hline \multirow[b]{3}{*}{$\gamma$} & \multirow[b]{3}{*}{ frac } & \multicolumn{6}{|c|}{ BP-ZDD } & \multicolumn{6}{|c|}{ BP-MIP } \\
\hline & & \multicolumn{3}{|c|}{$p$-range $[1,20]$} & \multicolumn{3}{|c|}{ p-range $[1,100]$} & \multicolumn{3}{|c|}{$p$-range $[1,20]$} & \multicolumn{3}{|c|}{ p-range $[1,100]$} \\
\hline & & $o p t$ & time & pricing & $o p t$ & time & pricing & $o p t$ & time & pricing & $o p t$ & time & pricing \\
\hline \multirow[t]{4}{*}{$0 \%$} & $1 / 4$ & 10 & 46.53 & 22.52 & 10 & 110.49 & 84.45 & 10 & 198.06 & 178.13 & 10 & 196.04 & 173.64 \\
\hline & $1 / 6$ & 10 & 45.18 & 23.37 & 10 & 131.71 & 92.07 & 10 & 264.59 & 245.20 & 10 & 522.65 & 487.14 \\
\hline & $1 / 8$ & 10 & 32.37 & 19.61 & 10 & 92.64 & 77.43 & 10 & 151.77 & 140.76 & 10 & 187.11 & 173.39 \\
\hline & $1 / 10$ & 10 & 26.67 & 16.23 & 10 & 82.25 & 69.33 & 10 & 128.86 & 119.10 & 10 & 150.62 & 139.62 \\
\hline \multirow[t]{4}{*}{$5 \%$} & $1 / 4$ & 10 & 59.80 & 34.03 & 10 & 122.19 & 95.69 & 10 & 242.77 & 221.96 & 10 & 249.59 & 228.55 \\
\hline & $1 / 6$ & 10 & 49.21 & 35.58 & 10 & 111.00 & 96.80 & 10 & 191.92 & 180.00 & 10 & 198.40 & 186.32 \\
\hline & $1 / 8$ & 10 & 45.83 & 31.70 & 10 & 92.89 & 79.87 & 0 & 1200.00 & 1198.89 & 10 & 448.60 & 436.73 \\
\hline & $1 / 10$ & 10 & 44.27 & 30.17 & 10 & 85.75 & 71.95 & 0 & 1200.00 & 1199.42 & 5 & 1001.39 & 994.70 \\
\hline \multirow[t]{4}{*}{$10 \%$} & $1 / 4$ & 10 & 79.40 & 50.77 & 10 & 150.20 & 124.36 & 10 & 288.28 & 264.71 & 10 & 318.59 & 293.93 \\
\hline & $1 / 6$ & 10 & 80.27 & 63.27 & 10 & 192.73 & 172.84 & 10 & 590.56 & 575.17 & 7 & 663.14 & 650.24 \\
\hline & $1 / 8$ & 10 & 81.27 & 61.00 & 10 & 190.52 & 163.42 & 2 & 1121.00 & 1112.07 & 1 & 1187.96 & 1182.99 \\
\hline & $1 / 10$ & 10 & 103.90 & 72.59 & 10 & 168.48 & 136.82 & 0 & 1200.00 & 1199.44 & 0 & 1200.00 & 1199.50 \\
\hline \multirow[t]{4}{*}{$15 \%$} & $1 / 4$ & 10 & 63.56 & 35.64 & 10 & 219.72 & 190.87 & 9 & 408.42 & 386.53 & 10 & 424.50 & 397.40 \\
\hline & $1 / 6$ & 10 & 80.48 & 52.08 & 10 & 289.97 & 251.38 & 8 & 755.99 & 732.60 & 3 & 1097.80 & 1073.34 \\
\hline & $1 / 8$ & 10 & 64.37 & 28.07 & 10 & 192.31 & 151.61 & 1 & 1139.44 & 1137.46 & 0 & 1200.00 & 1199.44 \\
\hline & $1 / 10$ & 10 & 65.08 & 25.41 & 10 & 138.91 & 101.48 & 0 & 1200.00 & 1199.50 & 0 & 1200.00 & 1199.56 \\
\hline \multirow[t]{4}{*}{$20 \%$} & $1 / 4$ & 10 & 83.32 & 57.07 & 10 & 336.67 & 300.37 & 10 & 449.94 & 422.62 & 10 & 655.45 & 620.39 \\
\hline & $1 / 6$ & 10 & 92.11 & 48.44 & 10 & 281.49 & 233.40 & 0 & 1200.00 & 1199.60 & 0 & 1200.00 & 1199.44 \\
\hline & $1 / 8$ & 10 & 58.05 & 24.77 & 10 & 170.46 & 126.25 & 1 & 1191.05 & 1190.24 & 0 & 1200.00 & 1199.53 \\
\hline & $1 / 10$ & 10 & 56.43 & 19.38 & 10 & 121.02 & 84.72 & 0 & 1200.00 & 1199.51 & 0 & 1200.00 & 1199.63 \\
\hline \multicolumn{2}{|c|}{ Overall } & 200 & 62.91 & 37.58 & 200 & 164.07 & 135.26 & 111 & 716.13 & 705.15 & 116 & 725.09 & 711.77 \\
\hline
\end{tabular}


Table 6: Computational results of the two B\&P algorithms on instances with $n=150$

\begin{tabular}{|c|c|c|c|c|c|c|c|c|c|c|c|c|c|}
\hline \multirow[b]{3}{*}{$\gamma$} & \multirow[b]{3}{*}{ frac } & \multicolumn{6}{|c|}{ BP-ZDD } & \multicolumn{6}{|c|}{ BP-MIP } \\
\hline & & \multicolumn{3}{|c|}{$p$-range $[1,20]$} & \multicolumn{3}{|c|}{$p$-range $[1,100]$} & \multicolumn{3}{|c|}{$p$-range $[1,20]$} & \multicolumn{3}{|c|}{ p-range $[1,100]$} \\
\hline & & $o p t$ & time & pricing & $o p t$ & time & pricing & opt & time & pricing & $o p t$ & time & pricing \\
\hline \multirow[t]{4}{*}{$0 \%$} & $1 / 4$ & 10 & 107.39 & 62.30 & 10 & 287.66 & 234.28 & 10 & 311.40 & 276.17 & 10 & 298.58 & 259.65 \\
\hline & $1 / 6$ & 10 & 112.37 & 72.19 & 10 & 338.97 & 273.99 & 10 & 420.35 & 386.11 & 10 & 762.51 & 703.91 \\
\hline & $1 / 8$ & 10 & 83.88 & 64.29 & 10 & 286.60 & 263.50 & 10 & 222.17 & 205.83 & 10 & 254.91 & 236.39 \\
\hline & $1 / 10$ & 10 & 70.49 & 54.39 & 10 & 243.58 & 225.52 & 10 & 186.21 & 171.92 & 10 & 212.08 & 196.64 \\
\hline \multirow[t]{4}{*}{$5 \%$} & $1 / 4$ & 10 & 108.90 & 66.42 & 10 & 304.21 & 257.63 & 10 & 407.44 & 366.83 & 10 & 422.32 & 379.29 \\
\hline & $1 / 6$ & 10 & 92.16 & 71.10 & 10 & 312.32 & 291.80 & 9 & 619.42 & 600.49 & 10 & 450.61 & 430.63 \\
\hline & $1 / 8$ & 10 & 79.88 & 61.00 & 10 & 271.83 & 253.45 & 0 & 1200.00 & 1199.19 & 0 & 1200.00 & 1199.29 \\
\hline & $1 / 10$ & 10 & 65.87 & 48.01 & 10 & 231.76 & 210.47 & 0 & 1200.00 & 1199.53 & 0 & 1200.00 & 1199.32 \\
\hline \multirow[t]{4}{*}{$10 \%$} & $1 / 4$ & 10 & 136.78 & 82.36 & 10 & 355.50 & 309.27 & 10 & 461.99 & 417.68 & 10 & 422.41 & 382.01 \\
\hline & $1 / 6$ & 10 & 135.55 & 109.52 & 10 & 607.62 & 577.89 & 0 & 1200.00 & 1198.58 & 6 & 1020.78 & 1005.84 \\
\hline & $1 / 8$ & 10 & 153.56 & 118.49 & 10 & 604.86 & 557.04 & 0 & 1200.00 & 1199.25 & 0 & 1200.00 & 1199.33 \\
\hline & $1 / 10$ & 10 & 156.47 & 101.74 & 10 & 498.29 & 429.09 & 0 & 1200.00 & 1199.14 & 0 & 1200.00 & 1199.36 \\
\hline \multirow[t]{4}{*}{$15 \%$} & $1 / 4$ & 10 & 164.96 & 114.57 & 10 & 654.63 & 602.49 & 10 & 600.37 & 546.83 & 9 & 676.27 & 630.98 \\
\hline & $1 / 6$ & 10 & 211.56 & 167.38 & 10 & 1006.05 & 929.47 & 4 & 1165.01 & 1133.67 & 0 & 1200.00 & 1188.77 \\
\hline & $1 / 8$ & 10 & 228.59 & 140.63 & 10 & 654.97 & 560.18 & 0 & 1200.00 & 1199.12 & 0 & 1200.00 & 1199.43 \\
\hline & $1 / 10$ & 10 & 202.36 & 115.29 & 10 & 485.35 & 393.37 & 0 & 1200.00 & 1199.07 & 0 & 1200.00 & 1199.50 \\
\hline \multirow[t]{4}{*}{$20 \%$} & $1 / 4$ & 10 & 243.43 & 188.72 & 4 & 980.14 & 935.49 & 9 & 859.23 & 805.61 & 10 & 912.05 & 853.43 \\
\hline & $1 / 6$ & 10 & 238.39 & 157.62 & 10 & 1052.19 & 951.14 & 1 & 1092.01 & 1091.07 & 0 & 1200.00 & 1199.30 \\
\hline & $1 / 8$ & 10 & 226.95 & 130.01 & 10 & 639.81 & 542.12 & 0 & 1200.00 & 1199.22 & 0 & 1200.00 & 1199.38 \\
\hline & $1 / 10$ & 10 & 171.78 & 95.56 & 10 & 359.53 & 251.86 & 0 & 1200.00 & 1199.10 & 0 & 1200.00 & 1199.35 \\
\hline \multicolumn{2}{|c|}{ Overall } & 200 & 149.57 & 101.08 & 194 & 508.79 & 452.50 & 93 & 857.28 & 839.72 & 95 & 871.63 & 853.09 \\
\hline
\end{tabular}

Table 7: Results of BP-ZDD for additional instances

\begin{tabular}{|c|c|c|c|c|c|c|c|c|c|c|c|c|c|}
\hline \multirow[b]{3}{*}{$\gamma$} & \multirow[b]{3}{*}{ frac } & \multicolumn{6}{|c|}{$n=180$} & \multicolumn{6}{|c|}{$n=150$} \\
\hline & & \multicolumn{3}{|c|}{$p$-range $[1,20]$} & \multicolumn{3}{|c|}{ p-range $[1,100]$} & \multicolumn{3}{|c|}{$p$-range $[1,200]$} & \multicolumn{3}{|c|}{$p$-range $[1,500]$} \\
\hline & & opt & time & pricing & opt & time & pricing & opt & time & pricing & opt & time & pricing \\
\hline \multirow[t]{4}{*}{$0 \%$} & $1 / 4$ & 10 & 344.04 & 142.71 & 10 & 771.38 & 574.02 & 10 & 554.88 & 461.03 & 2 & 1182.41 & 1108.91 \\
\hline & $1 / 6$ & 10 & 337.70 & 178.99 & 10 & 965.65 & 690.92 & 10 & 776.64 & 552.76 & 0 & 1200.00 & 1001.85 \\
\hline & $1 / 8$ & 10 & 213.44 & 176.42 & 10 & 615.19 & 569.55 & 10 & 435.96 & 408.85 & 9 & 1058.38 & 1028.34 \\
\hline & $1 / 10$ & 10 & 179.88 & 156.05 & 10 & 608.43 & 577.72 & 10 & 373.80 & 354.90 & 10 & 827.55 & 807.53 \\
\hline \multirow[t]{4}{*}{$5 \%$} & $1 / 4$ & 10 & 349.83 & 147.86 & 10 & 835.84 & 611.28 & 10 & 558.37 & 458.03 & 0 & 1200.00 & 1177.87 \\
\hline & $1 / 6$ & 10 & 249.39 & 187.41 & 10 & 744.31 & 680.39 & 10 & 591.52 & 558.25 & 0 & 1200.00 & 1200.00 \\
\hline & $1 / 8$ & 10 & 199.82 & 159.45 & 10 & 658.74 & 624.39 & 10 & 489.28 & 467.03 & 0 & 1200.00 & 1196.15 \\
\hline & $1 / 10$ & 10 & 197.08 & 152.98 & 10 & 587.63 & 542.07 & 10 & 433.70 & 404.63 & 0 & 1200.00 & 1194.26 \\
\hline \multirow[t]{4}{*}{$10 \%$} & $1 / 4$ & 10 & 424.99 & 198.87 & 7 & 1037.50 & 820.74 & 10 & 880.18 & 741.25 & 1 & 1105.82 & 1082.74 \\
\hline & $1 / 6$ & 10 & 348.25 & 281.94 & 1 & 1084.11 & 1052.73 & 4 & 1175.03 & 1135.05 & 0 & 1200.00 & 1195.57 \\
\hline & $1 / 8$ & 10 & 440.08 & 340.55 & 0 & 1200.00 & 1129.72 & 6 & 1168.89 & 1091.73 & 0 & 1200.00 & 1188.47 \\
\hline & $1 / 10$ & 10 & 565.02 & 330.88 & 1 & 1082.24 & 890.34 & 10 & 904.26 & 769.31 & 0 & 1200.00 & 1135.04 \\
\hline \multirow[t]{4}{*}{$15 \%$} & $1 / 4$ & 10 & 423.59 & 209.02 & 1 & 1092.79 & 991.88 & 0 & 1200.00 & 1141.92 & 0 & 1200.00 & 1200.00 \\
\hline & $1 / 6$ & 10 & 709.96 & 538.11 & 1 & 1087.15 & 1003.63 & 0 & 1200.00 & 1130.43 & 0 & 1200.00 & 1200.00 \\
\hline & $1 / 8$ & 9 & 866.95 & 348.07 & 0 & 1200.00 & 901.36 & 1 & 1194.90 & 1031.59 & 0 & 1200.00 & 1164.33 \\
\hline & $1 / 10$ & 10 & 776.02 & 324.79 & 1 & 1084.03 & 771.81 & 10 & 936.51 & 724.93 & 0 & 1200.00 & 1116.85 \\
\hline \multirow[t]{4}{*}{$20 \%$} & $1 / 4$ & 10 & 652.25 & 367.11 & 1 & 1106.16 & 1056.38 & 0 & 1200.00 & 1182.59 & 0 & 1200.00 & 1200.00 \\
\hline & $1 / 6$ & 10 & 1055.02 & 471.28 & 1 & 1089.34 & 895.27 & 0 & 1200.00 & 1082.14 & 0 & 1200.00 & 1200.00 \\
\hline & $1 / 8$ & 10 & 863.69 & 316.90 & 0 & 1200.00 & 870.55 & 3 & 1191.56 & 942.89 & 0 & 1200.00 & 1166.88 \\
\hline & $1 / 10$ & 10 & 775.53 & 266.71 & 7 & 1028.15 & 596.23 & 10 & 743.34 & 513.92 & 0 & 1200.00 & 1084.69 \\
\hline \multicolumn{2}{|c|}{ Overall } & 199 & 498.63 & 264.81 & 101 & 953.93 & 792.55 & 134 & 860.44 & 757.66 & 22 & 1168.71 & 1132.47 \\
\hline
\end{tabular}




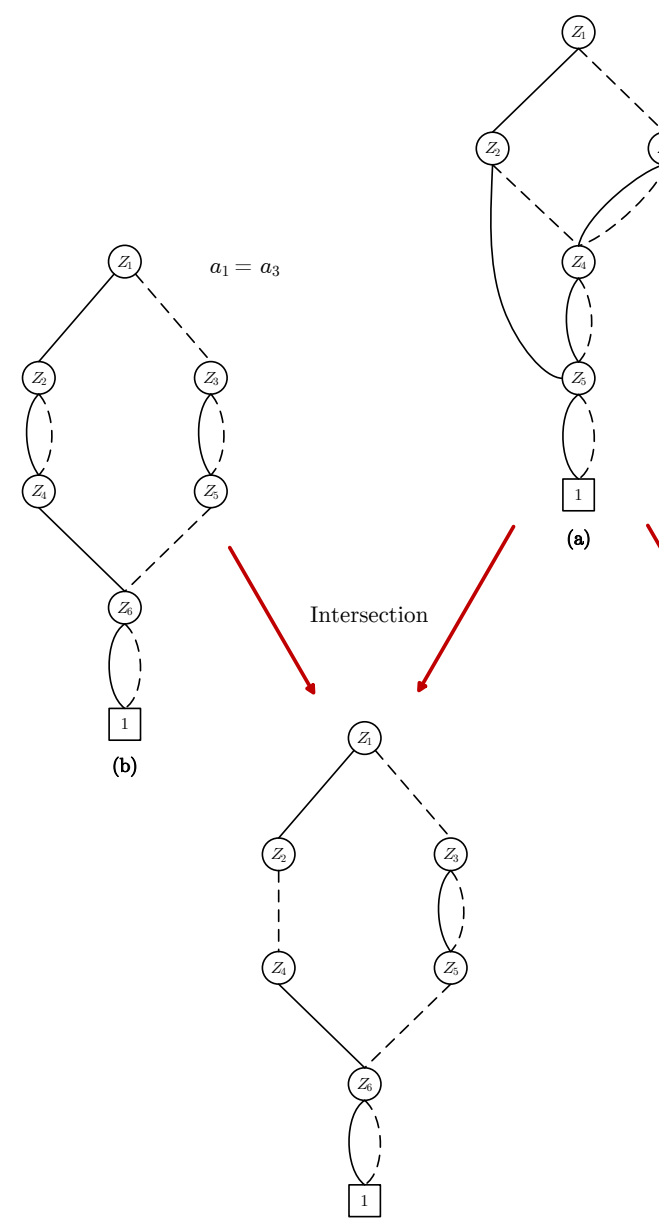

(d)

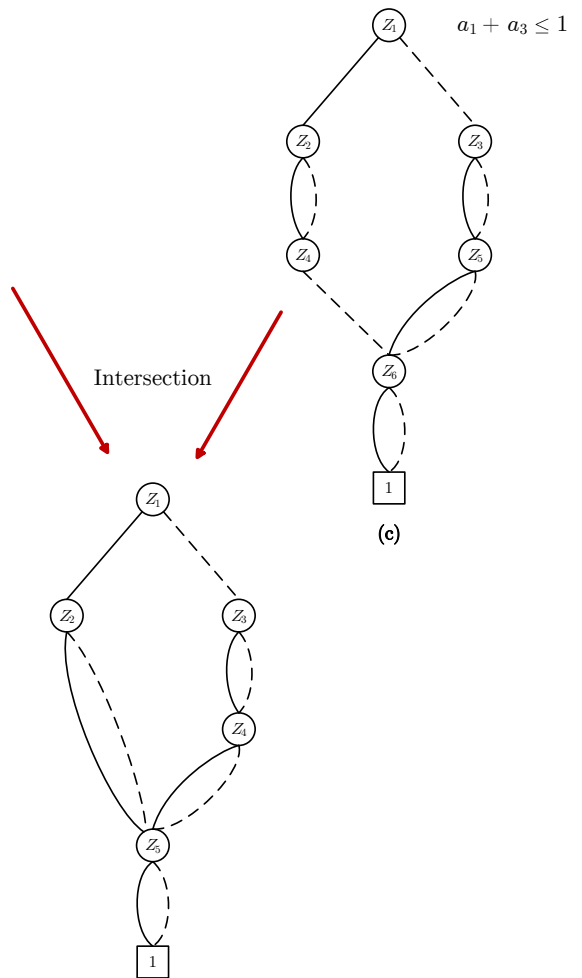

(e)

Figure 3: Construction of the ZDDs for the child nodes in the example instance (branch on jobs 1 and 3 )

with relatively large $\Gamma$ values and shorter deadlines. For instances of size 30 , the intuitive formulation already fails to solve 74 out of 400 instances, and its overall run time is orders of magnitude higher than for the B\&P algorithms reported below. Due to this poor computational performance, we will not further include this formulation in the comparison for larger instances.

Two B\&P algorithms are implemented and compared for $n=60,90,120$ and 150. The global routine is the same, the difference resides in the pricing solver. We denote the $\mathrm{B} \& \mathrm{P}$ algorithm with the ZDD-based pricing solver as BP-ZDD, while BP-MIP refers to the use of a MIP solver for formulation (9) for pricing. In BP-ZDD the branching constraints are imposed on the ZDDs as described in Section 5.3, while in BP-MIP they are added to formulation (9). The computational comparison of BP-ZDD and BP-MIP is summarized in Tables 3 to 6 . Since both B\&P algorithms follow the same overall routine and differ only in the pricing method, the average run time spent 
on the pricing procedures is included separately in the columns labeled pricing (in seconds).

Overall, BP-ZDD yields a consistently better performance than BP-MIP, solving all instances from size 60 to size 120 and only failing to solve six instances with size 150. BP-MIP already starts to experience difficulties for size 60 . The performance gradually worsens with increasing $n$, with less than half of the instances with $n=150$ solved to guaranteed optimality. The reason why BP-ZDD fails on six instances is because the size of the ZDDs becomes very large, with number of nodes close to one million, thus the construction of ZDDs and branching are no longer easy to handle and it takes longer to trace a longest path in those ZDDs. When BP-MIP fails, it usually gets stuck with a pricing problem. The benefit of applying ZDDs for pricing is obvious in the smaller instances $(n=60)$ : the average run time for pricing in BP-ZDD is lower than BP-MIP by roughly two orders of magnitude. For larger instances, the actual difference in run times cannot be accurately measured as BP-MIP failed for many instances, but it is clear that BP-ZDD achieves a superior performance thanks to its more efficient pricing solver.

The value of $\Gamma$ significantly influences the difficulty of the instances. For both algorithms, the larger the $\Gamma$ value, the longer it takes for an algorithm to solve an instance. For BP-MIP, the number of solved instances clearly decreases as the value of $\Gamma$ grows, and so the consideration of robustness, which is reflected in $\Gamma$, is one of the main factors of difficulty of an instance.

BP-ZDD and BP-MIP have a very different dependence on the deadline. As mentioned before, the pricing problem is a robust variant of the knapsack problem. Since a larger deadline means that more jobs can be assigned to one individual machine, this increases the difficulty in BP-ZDD due to the larger ZDDs, with longer paths and more nodes, which explains why BP-ZDD cannot solve all instances in Table 6 with the largest $\Gamma$ and highest frac. For BP-MIP exactly the opposite occurs: it can still solve most of the instances with high frac but struggles with lower frac. This phenomenon goes against the trend for knapsack problems; one possible reason is that the extra constraints and variables in formulation (9) significantly change its structure compared to a general knapsack problem.

The different ranges of processing times do not have much impact on the CPU time and the number of solved instances for BP-MIP. For BP-ZDD, by contrast, a wider range coincides with more states in the ZDDs, which increases the size of the ZDDs and makes the manipulation of ZDDs less efficient. Tables 3 to 6 show that the average pricing time for range $[1,20]$ is only a 
fraction of the results for the wider range $[1,100]$ for BP-ZDD, and its average run time in any setting with time range $[1,20]$ never exceeds 250 seconds.

In order to explore the limits of BP-ZDD, we have tested the procedure on two additional data sets. The first set of instances has more jobs $(n=180)$ but similar processing-time ranges as before $([1,20]$ and $[1,100])$, while the second set has $n=150$ but durations are generated from the wider

ranges $[1,200]$ and $[1,500]$. The results are shown in Table 7. For $n=180$, BP-ZDD still solves 199 out of 200 instances with range $[1,20]$, and about half of the instances with range $[1,100]$, mainly with small $\Gamma$ values. For $n=150$ but with wider ranges, BP-ZDD experiences more difficulties, and fails almost completely for range $[1,500]$, with only one instance with nonzero $\Gamma$ value solved. This confirms that wider processing-time ranges entail greater difficulty for BP-ZDD.

\subsection{Results for bin packing instances}

The deterministic variant of RMAP coincides with the classic BPP. Since our procedure solves a more general problem variant, it can also solve the conventional BPP. In order to validate the performance of our method, we therefore also test on some BPP benchmarks, and compare with several methods for the BPP. We have run our procedure on all the sets that are fully tested in Delorme et al. (2016), including the set "Falkenauer T" from Falkenauer (1996) with $n$ from 60 to 501 and bin capacity $c=1000$, set "Scholl 3" of 10 difficult instances from Scholl et al. (1997) with $n=200, c=100000$ and item sizes generated from [20 000,35000], set "Wäscher" of 17 hard instances from Wäscher and Gau (1996) with $n$ from 57 to 239 and $c=10000$, sets "Schwerin 1" and "Schwerin 2" from Schwerin and Wäscher (1997), with 100 instances both for $n=100$ and for 120, and set "Hard28," which contains 28 hard instances from Schoenfield (2002), with sizes from 160 to 200. The data are obtained from the BPPLIB (Delorme et al., 2017).

Three algorithms for the BPP are chosen for comparison, which are the well-known B\&B algorithm BISON from Scholl et al. (1997), the classic B\&P algorithm proposed by Vance et al. (1994), denoted as VANCE, which also uses the Ryan and Foster branching rule, and the branch-and-cutand-price algorithm by Belov and Scheithauer (2006), denoted as BELOV. The computational results for these benchmark algorithms are taken from the latest BPP review by Delorme et al. (2016), where the experiments are executed on a quad-core Intel Xeon CPU at 3.10 GHz with 8 GB RAM using only one thread on one core. Correspondingly, BP-ZDD is also set to run with only 
Table 8: Number of the BPP instances solved by different methods in less than one minute

\begin{tabular}{lccccc}
\hline Set & Total & BISON & VANCE & BELOV & BP-ZDD \\
\hline Falkenauer T & 80 & 42 & 76 & 57 & 52 \\
Scholl 3 & 10 & 3 & 10 & 10 & 0 \\
Wäscher & 17 & 10 & 6 & 17 & 6 \\
Schwerin 1 & 100 & 100 & 100 & 100 & 100 \\
Schwerin 2 & 100 & 63 & 100 & 100 & 100 \\
Hard28 & 28 & 0 & 11 & 28 & 11 \\
\hline Overall & 335 & 218 & 303 & 312 & 269 \\
\hline
\end{tabular}

one thread of one core.

For BP-ZDD, we simply set $\Gamma$ and the processing-time deviations to zero. The results of the four methods on these instance sets are reported in Table 8 for a time limit of one minute. It can be seen that BP-ZDD solves more instances than BISON overall, but fewer than VANCE and BELOV. It struggles especially for instances with large number of items and wide range of sizes, such as Scholl 3, Wäscher and Hard28. For some data sets, it matches the performance of VANCE, which has a similar algorithmic structure. The state-of-the-art BELOV algorithm is able to find guaranteed optimal solutions to more instances. BELOV has the advantage of generating additional constraints to cut off non-integral solutions. We have not applied such enhancements in our procedure, because the generated cuts and the dual variables entailed by the cuts generally invalidate DP for the pricing problem (Belov and Scheithauer, 2006), while we see no efficient implicit enumeration method that could solve the corresponding pricing problem in RMAP Belov and Scheithauer, 2006, use B\&B for their pricing problem). Since the procedure BP-ZDD has been developed for a more general problem setting, we conclude that it has an adequate competitive performance on the conventional BPP instances of moderate size.

\subsection{Sensitivity analysis}

The budget of uncertainty $\Gamma$ is the key parameter of our model. In this section we examine its influence on the optimal objective value, and following up on our discussion in Section 6.2 we also zoom in on the impact of $\Gamma$ on the difficulty of the instances, for which the size of the ZDD at the root node is a direct indicator. We generate 10 instances of 120 jobs, fixing the duration deviations at $30 \%$ of the processing times (rounded to the higher integer), and frac $=1 / 10$. For a wide range of $\Gamma$ values, we compute the average objective value (number of machines) and the size of the ZDD 


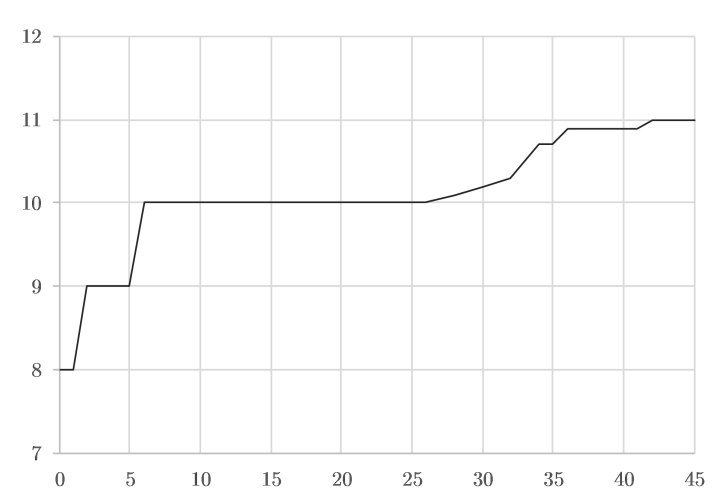

(a) Average number of machines as a function of $\Gamma$

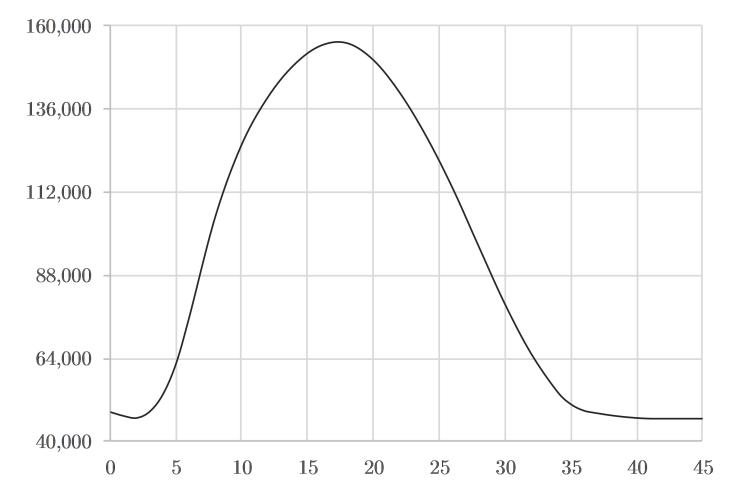

(b) Average size of the ZDD at the root node as a function of $\Gamma$

Figure 4: The impact of $\Gamma$ on the objective value and on the difficulty of the instances

at the root node. The results are shown in Figure 4.

Figure 4 (a) depicts the number of machines dependent on $\Gamma$. The curve is monotone nondecreasing overall, as more machines or higher cost is incurred to guarantee a stronger robustness. The curve starts at $\Gamma=0$ (no protection provided), where eight machines are needed on average. The curve reaches its maximum value of 11 at $\Gamma=42$, and any $\Gamma \geq 42$ simply corresponds to the same worst case. The curve displays several plateaus, which is a consequence of the slackness provided in the gap between the optimal LP and integral solution. Within these plateaus, the objective value is insensitive to $\Gamma$, where a relatively larger $\Gamma$ could be chosen for higher robustness.

Figure 4(b) plots the average size of the root-node ZDD as a function of $\Gamma$; a bell-shaped pattern occurs. In the left part of the graph, the average size climbs with increasing $\Gamma$, reaching a peak at around 155, 000 for $\Gamma=18$. Here, larger $\Gamma$ results in more states in the ZDDs, and generates more unique paths with non-equivalent nodes that cannot be suppressed. As $\Gamma$ increases beyond value 18, the plot goes down again, because more jobs are forced to take on their worst-case duration along each path, and thus more similar paths are generated with more equivalent nodes that can be merged in the ZDDs. When $\Gamma$ reaches a critical value where all jobs are forced into their worst-case duration, the size of the ZDD no longer changes with increasing $\Gamma$. At the two ends of the curve, the average sizes of the ZDDs are very close to each other, which is not illogical: both $\Gamma$ values essentially represent deterministic cases, either the nominal case or the worst case. 


\section{Conclusions}

In this work, we have considered a time-driven scheduling problem in a parallel machine environment. This machine availability problem minimizes the number of identical machines that are necessary to complete the job set before a given deadline. We have studied this problem in a context of uncertainty, leading to the robust machine availability problem RMAP, which uses an uncertainty set for the job durations following Bertsimas and Sim (2004), with a budget of uncertainty limiting the number of deviating job durations. The deterministic version of the problem coincides with the BPP, and thereby we are also one of the first to study robust bin packing.

An intuitive formulation for RMAP is presented, followed by a set covering formulation and a $\mathrm{B} \& \mathrm{P}$ procedure for better computational performance. We introduce ZDDs for solving the pricing problem to tackle the difficulty entailed by the robustness considerations and by the extra constraints imposed by branching decisions. Two B\&P algorithms are tested and compared, where pricing is done using ZDDs and using a generic MIP solver, respectively. Our computational results show that the B\&P algorithm with ZDDs has superior performance. Experiments on classic bin packing instances confirm the adequate functioning of the proposed algorithm.

Further research in this area may include the study of different objective functions, such as makespan minimization or the sum of weighted completion times; to this respect, it should be noted that the current paper also contributes to the development of a solution method for a corresponding robust variant of $P \| C_{\max }$, which could be solved using RMAP as a subproblem in a binary search procedure, similarly to Dell'Amico et al. (2008). There are also possible branching schemes for RMAP that could preserve a certain structure for the pricing subproblem such that that problem can still be solved by a DP algorithm after branching (e.g., Chen and Powell, 1999, Bulhões et al., 2017), which deserve further investigation. Other opportunities for studying richer scheduling models are legion, such as the introduction of precedence constraints or the inclusion of multiple resource categories.

\section{References}

Akers, S. B. (1978). Binary decision diagrams. IEEE Transactions on Computers, 27(6):509-516. 
Belov, G. and Scheithauer, G. (2006). A branch-and-cut-and-price algorithm for one-dimensional stock cutting and two-dimensional two-stage cutting. European Journal of Operational Research, 171(1):85-106.

Ben-Tal, A. and Nemirovski, A. (1998). Robust convex optimization. Mathematics of Operations Research, 23(4):769-805.

Ben-Tal, A. and Nemirovski, A. (1999). Robust solutions of uncertain linear programs. Operations Research Letters, 25(1):1-13.

Ben-Tal, A. and Nemirovski, A. (2000). Robust solutions of linear programming problems contaminated with uncertain data. Mathematical Programming, 88(3):411-424.

Bergman, D., Cire, A. A., van Hoeve, W.-J., and Hooker, J. N. (2016). Discrete optimization with decision diagrams. INFORMS Journal on Computing, 28(1):47-66.

Bertsimas, D., Brown, D. B., and Caramanis, C. (2011). Theory and applications of robust optimization. SIAM Review, 53(3):464-501.

Bertsimas, D. and Sim, M. (2003). Robust discrete optimization and network flows. Mathematical Programming, 98(1-3):49-71.

Bertsimas, D. and Sim, M. (2004). The price of robustness. Operations Research, 52(1):35-53.

Bertsimas, D. and Thiele, A. (2006). Robust and data-driven optimization: modern decision making under uncertainty. In Models, Methods, and Applications for Innovative Decision Making, pages 95-122. INFORMS.

Bougeret, M., Pessoa, A. A., and Poss, M. (2016). Robust scheduling with budgeted uncertainty. HAL, open archives, hal-01345283. Available at https://hal.archives-ouvertes. fr/hal-01345283.

Bruni, M., Pugliese, L. D. P., Beraldi, P., and Guerriero, F. (2017). An adjustable robust optimization model for the resource-constrained project scheduling problem with uncertain activity durations. Omega, 71:66-84. 
Bulhões, T., Sadykov, R., Uchoa, E., and Subramanian, A. (2017). On the exact solution of a large class of parallel machine scheduling problems. In Proceedings of MISTA 2017, the 8th Multidisciplinary International Conference on Scheduling: Theory and Applications, pages 325328, Kuala Lumpur, Malaysia.

Chen, Z.-L. and Powell, W. B. (1999). Solving parallel machine scheduling problems by column generation. INFORMS Journal on Computing, 11(1):78-94.

Chopra, S. and Meindl, P. (2013). Supply Chain Management: Strategy, Planning, And Operation. Pearson.

Coughlan, E. T., Lübbecke, M. E., and Schulz, J. (2015). A branch-price-and-cut algorithm for multi-mode resource leveling. European Journal of Operational Research, 245(1):70-80.

Daniels, R. L. and Kouvelis, P. (1995). Robust scheduling to hedge against processing time uncertainty in single-stage production. Management Science, 41(2):363-376.

Dell'Amico, M., Iori, M., Martello, S., and Monaci, M. (2008). Heuristic and exact algorithms for the identical parallel machine scheduling problem. INFORMS Journal on Computing, 20(3):333344.

Dell'Amico, M. and Martello, S. (1995). Optimal scheduling of tasks on identical parallel processors. ORSA Journal on Computing, 7(2):191-200.

Dell'Amico, M. and Martello, S. (2005). A note on exact algorithms for the identical parallel machine scheduling problem. European Journal of Operational Research, 160(2):576-578.

Delorme, M., Iori, M., and Martello, S. (2016). Bin packing and cutting stock problems: Mathematical models and exact algorithms. European Journal of Operational Research, 255(1):1-20.

Delorme, M., Iori, M., and Martello, S. (2017). BPPLIB: A library for bin packing and cutting stock problems. Optimization Letters, to appear.

Demeulemeester, E. (1995). Minimizing resource availability costs in time-limited project networks. Management Science, 41(10):1590-1598. 
Dyckhoff, H. (1981). A new linear programming approach to the cutting stock problem. Operations Research, 29(6):1092-1104.

Easa, S. M. (1989). Resource leveling in construction by optimization. Journal of Construction Engineering and Management, 115(2):302-316.

El Ghaoui, L. and Lebret, H. (1997). Robust solutions to least-squares problems with uncertain data. SIAM Journal on Matrix Analysis and Applications, 18(4):1035-1064.

El Ghaoui, L., Oustry, F., and Lebret, H. (1998). Robust solutions to uncertain semidefinite programs. SIAM Journal on Optimization, 9(1):33-52.

Falkenauer, E. (1996). A hybrid grouping genetic algorithm for bin packing. Journal of Heuristics, $2(1): 5-30$.

Farley, A. A. (1990). A note on bounding a class of linear programming problems, including cutting stock problems. Operations Research, 38(5):922-923.

Garey, M. R. and Johnson, D. S. (1979). Computers and Intractability: A Guide to the Theory of NP-completeness. W. H. Freeman and Company, New York.

Gilmore, P. C. and Gomory, R. E. (1961). A linear programming approach to the cutting-stock problem. Operations Research, 9(6):849-859.

Graves, S. C. (1981). A review of production scheduling. Operations Research, 29(4):646-675.

Hans, E. (2001). Resource loading by branch-and-price techniques. PhD thesis, University of Twente, The Netherlands.

Harris, R. B. (1990). Packing method for resource leveling (pack). Journal of Construction Engineering and Management, 116(2):331-350.

Herroelen, W. and Leus, R. (2005). Project scheduling under uncertainty: Survey and research potentials. European Journal of Operational Research, 165(2):289-306.

Hooker, J. N. (2013). Decision diagrams and dynamic programming. In International Conference on AI and OR Techniques in Constraint Programming for Combinatorial Optimization Problems, pages 94-110. Springer. 
IBM (2015). IBM ILOG CPLEX Optimization Studio CPLEX User's Manual. Version 12 Release 6, Available at https://www.ibm.com/support/knowledgecenter/SSSA5P_12.6.3/ilog. odms.studio.help/pdf/usrcplex.pdf.

Iwashita, H. and Minato, S. (2013). Efficient top-down ZDD construction techniques using recursive specifications. TCS Technical reports TCS-TR-A-13-69, Hokkaido University, Division of Computer Science.

Kasperski, A. (2005). Minimizing maximal regret in the single machine sequencing problem with maximum lateness criterion. Operations Research Letters, 33(4):431-436.

Knuth, D. (2009). The Art of Computer Programming, Volume 4, Fascicle 1: Bitwise Tricks E Techniques; Binary Decision Diagrams. Addison-Wesley Professional, 12th edition.

Kouvelis, P., Daniels, R. L., and Vairaktarakis, G. (2000). Robust scheduling of a two-machine flow shop with uncertain processing times. IIE Transactions, 32(5):421-432.

Kouvelis, P. and Yu, G. (1997). Robust Discrete Optimization and Its Applications. Kluwer, Dordrecht.

Kowalczyk, D. and Leus, R. (2018). A branch-and-price algorithm for parallel machine scheduling using ZDDs and generic branching. INFORMS Journal on Computing, to appear.

Lee, C.-Y. (1959). Representation of switching circuits by binary-decision programs. Bell Labs Technical Journal, 38(4):985-999.

Leu, S.-S., Yang, C.-H., and Huang, J.-C. (2000). Resource leveling in construction by genetic algorithm-based optimization and its decision support system application. Automation in Construction, 10(1):27-41.

Leus, R. and Herroelen, W. (2004). Stability and resource allocation in project planning. IIE Transactions, 36(7):667-682.

Leus, R. and Herroelen, W. (2007). Scheduling for stability in single-machine production systems. Journal of Scheduling, 10(3):223-235. 
Lopes, M. J. P. and Valério De Carvalho, J. M. (2007). A branch-and-price algorithm for scheduling parallel machines with sequence dependent setup times. European Journal of Operational Research, 176(3):1508-1527.

Lu, C.-C., Lin, S.-W., and Ying, K.-C. (2012). Robust scheduling on a single machine to minimize total flow time. Computers \& Operations Research, 39(7):1682-1691.

Minato, S. (1993). Zero-suppressed BDDs for set manipulation in combinatorial problems. In Proceedings of the 30th international Design Automation Conference, pages 272-277. ACM.

Minato, S. (2001). Zero-suppressed BDDs and their applications. International Journal on Software Tools for Technology Transfer, 3(2):156-170.

Möhring, R. H. (1984). Minimizing costs of resource requirements in project networks subject to a fixed completion time. Operations Research, 32(1):89-120.

Mokotoff, E. (2004). An exact algorithm for the identical parallel machine scheduling problem. European Journal of Operational Research, 152(3):758-769.

Morrison, D. R., Sewell, E. C., and Jacobson, S. H. (2016). Solving the pricing problem in a branch-and-price algorithm for graph coloring using zero-suppressed binary decision diagrams. INFORMS Journal on Computing, 28(1):67-82.

Mula, J., Poler, R., Garcia-Sabater, J., and Lario, F. C. (2006). Models for production planning under uncertainty: A review. International Journal of Production Economics, 103(1):271-285.

Pessoa, A. A., Di Puglia, P., Guerriero, F., and Poss, M. (2015). Robust constrained shortest path problems under budgeted uncertainty. Networks, 66(2):98-111.

Pessoa, A. A., Uchoa, E., Poggi de Aragão, M., and Rodrigues, R. (2010). Exact algorithm over an arc-time-indexed formulation for parallel machine scheduling problems. Mathematical Programming Computation, 2(3):259-290.

Pferschy, U. and Schauer, J. (2009). The knapsack problem with conflict graphs. Journal of Graph Algorithms and Applications, 13(2):233-249.

Pinedo, M. (2015). Scheduling: Theory, Algorithms, and Systems. Springer, 5th edition. 
Ranjbar, M., Davari, M., and Leus, R. (2012). Two branch-and-bound algorithms for the robust parallel machine scheduling problem. Computers $\&$ Operations Research, 39(7):1652-1660.

Rhee, W. T. and Talagrand, M. (1993). On line bin packing with items of random size. Mathematics of Operations Research, 18(2):438-445.

Rodrigues, S. B. and Yamashita, D. S. (2010). An exact algorithm for minimizing resource availability costs in project scheduling. European Journal of Operational Research, 206(3):562-568.

Ryan, D. M. and Foster, B. A. (1981). An integer programming approach to scheduling. Computer Scheduling of Public Transport Urban Passenger Vehicle and Crew Scheduling, pages 269-280.

Schoenfield, J. E. (2002). Fast, exact solution of open bin packing problems without linear programming. US Army Space and Missile Defense Command Technical Report, Huntsville, Alabama, USA.

Scholl, A., Klein, R., and Jürgens, C. (1997). Bison: A fast hybrid procedure for exactly solving the one-dimensional bin packing problem. Computers \& Operations Research, 24(7):627-645.

Schwerin, P. and Wäscher, G. (1997). The bin-packing problem: A problem generator and some numerical experiments with FFD packing and MTP. International Transactions in Operational Research, 4(5-6):377-389.

Soyster, A. L. (1973). Convex programming with set-inclusive constraints and applications to inexact linear programming. Operations Research, 21(5):1154-1157.

Valério De Carvalho, J. M. (2002). LP models for bin packing and cutting stock problems. European Journal of Operational Research, 141(2):253-273.

van den Akker, J. M., Hoogeveen, J. A., and van de Velde, S. L. (1999). Parallel machine scheduling by column generation. Operations Research, 47(6):862-872.

Vance, P. H. (1998). Branch-and-price algorithms for the one-dimensional cutting stock problem. Computational Optimization and Applications, 9(3):211-228. 
Vance, P. H., Barnhart, C., Johnson, E. L., and Nemhauser, G. L. (1994). Solving binary cutting stock problems by column generation and branch-and-bound. Computational Optimization and Applications, 3(2):111-130.

Vanderbeck, F. (1999). Computational study of a column generation algorithm for bin packing and cutting stock problems. Mathematical Programming, 86(3):565-594.

Vanderbeck, F. (2000). Exact algorithm for minimising the number of setups in the one-dimensional cutting stock problem. Operations Research, 48(6):915-926.

Wäscher, G. and Gau, T. (1996). Heuristics for the integer one-dimensional cutting stock problem: A computational study. OR Spectrum, 18(3):131-144.

Wiest, J. D., Levy, F. K., et al. (1969). Management guide to PERT/CPM. NJ, Prentice-Hall.

Wullink, G., Gademann, A., Hans, E. W., and van Harten, A. (2004). Scenario-based approach for flexible resource loading under uncertainty. International Journal of Production Research, 42(24):5079-5098.

Xu, X., Cui, W., Lin, J., and Qian, Y. (2013). Robust makespan minimisation in identical parallel machine scheduling problem with interval data. International Journal of Production Research, 51(12):3532-3548.

Xu, X., Lin, J., and Cui, W. (2014). Hedge against total flow time uncertainty of the uniform parallel machine scheduling problem with interval data. International Journal of Production Research, 52(19):5611-5625.

Yang, J. and Yu, G. (2002). On the robust single machine scheduling problem. Journal of Combinatorial Optimization, 6(1):17-33. 